\title{
Conmensurabilidad empírica entre teorías inconmensurables: el caso del flogisto"
}

\author{
Empirical Commensurability \\ between Incommensurable Theories: The Phlogiston Case
}

María Caamaño

\begin{abstract}
Resumen
El presente trabajo pretende dar respuesta a la demanda, abiertamente hecha por Kuhn (1976) y Feyerabend (1977), así como por los impulsores de la corriente estructuralista, Balzer, Moulines \& Sneed (1987), sobre la necesidad de analizar estructuralmente casos históricos de teorías inconmensurables para llegar a precisar formalmente y dar soporte empírico a la noción de inconmensurabilidad. El trabajo se estructura en tres partes: en la primera, se aclara el tipo de entidad teórica que conformarían las teorías del flogisto y del oxígeno; en la segunda, se presentan sus correspondientes reconstrucciones estructurales y finalmente, en la tercera, se desarrolla un análisis de las relaciones interteóricas entre la teoría del flogisto y la del oxígeno, mostrando cómo su modo de relacionarse satisface las condiciones establecidas en las definiciones de reducción ontológica débil y de inconmensurabilidad.
\end{abstract}

Palabras clave: inconmensurabilidad - teoría del flogisto - reconstrucción estructural - reducción ontológica

\begin{abstract}
This work constitutes an attempt to meet Kuhn's (1976) and Feyerabend's (1977) request, also shared by the main proponents of the structuralist meta-theory, Balzer, Moulines and Sneed (1987), for a structural analysis of incommensurable theories, so that it becomes possible to formally specify and empirically support the notion of incommensurability. The paper is structured in three parts: first, the kind of theoretical entity corresponding to the phlogiston and oxygen theories is determined; second, their respective structural reconstructions are presented, and, finally, an analysis of both theories' intertheoretical relations is developed, showing that they satisfy the definitions of weak ontological reduction and incommensurability.
\end{abstract}

Keywords: incommensurability - phlogiston theory - structural reconstruction - ontological reduction

* Recibido: 14 Enero 2011. Aceptado en versión revisada: 21 Marzo 2011.

${ }^{\dagger}$ Departamento de Filosofía, Facultad de Filosofía y Letras, Universidad de Valladolid. Para contactar al autor, por favor escriba a: mariac@fyl.uva.es.

‡Estoy en deuda con José A. Díez por su extraordinaria perspicacia y paciencia en la revisión de muchas versiones previas de este artículo. También estoy enormemente agradecida a C. Ulises Moulines por proporcionarme claras directrices con las que abordar el tratamiento estructuralista del caso del flogisto. Agradezco, asimismo, a Pablo Lorenzano sus valiosos comentarios sobre mi trabajo durante el VI Encuentro Iberoamericano de Metateoría Estructuralista celebrado en Auxerre (Francia, 2008). Este trabajo ha sido financiado por el Ministerio de Educación de España, la Fundación Fulbright y con cargo a los proyectos "Lenguaje y pensamiento: dependencia contextual" (FFI2008-06421-CO2-02/FISO) y "Actitudes cognitivas y la justificación del conocimiento" (FFI2009-08828), ambos subvencionados por el Ministerio de Ciencia e Innovación español.

Metatheoria 1(2)(2011): 131-166. ISSN 1853-2322.

(C) Editorial de la Universidad Nacional de Tres de Febrero. Publicado en la República Argentina. 


\section{Aclaraciones preliminares \\ 1.1. Enfoque, objetivos y estructura del trabajo}

Este trabajo aborda el problema de la inconmensurabilidad de las teorías científicas, centrándose en un episodio histórico concreto propuesto por T.S. Kuhn como un caso paradigmático de inconmensurabilidad. Recordemos que la noción de inconmensurabilidad fue introducida simultánea e independientemente por Thomas S. Kuhn y Paul K. Feyerabend en 1962, con objeto de caracterizar la relación entre teorías científicas rivales. ${ }^{1}$ La relación es caracterizada por cada autor de modo parcialmente divergente, aunque ambos coinciden en presentarla como una forma radical de disparidad conceptual entre teorías que se aplican a un mismo dominio de investigación. La radicalidad de la disparidad conceptual se debería no solo a que las teorías estarían constituidas por conceptos diferentes, sino también a que las condiciones de formación de tales conceptos serían incompatibles. De acuerdo con ambos autores, ello se manifiesta en la incompatibilidad entre las reglas de uso de los términos que transmiten dichos conceptos. La inconmensurabilidad entre teorías científicas rivales quedaría patente no solo en la imposibilidad de traducir mutuamente los lenguajes que expresan tales teorías, sino también en la imposibilidad de ampliar uno de los lenguajes de modo que incorpore el otro.

La defensa de la tesis de la inconmensurabilidad, por parte de Kuhn y Feyerabend, ha ido pareja tanto a su rechazo de los modelos verificacionista y falsacionista de la relación entre teorías sucesivas, como a su reivindicación de una versión radical de la tesis de la carga teórica de la observación. La dependencia teórica de toda observación, entendida como dependencia de toda observación con respecto a la teoría para la que cuenta como observación, implica una relativización de toda observación al contexto teórico en el que es tenida en cuenta. Lo anterior conlleva tres consecuencias estrechamente conectadas: 1) el rechazo de la distinción entre enunciados teóricos y enunciados observacionales, 2) la asunción de un holismo semántico radical, conforme al cual el significado de un término depende del contexto teórico en el que ocurre, y 3 ) una inconmensurabilidad semántica radical, derivada del alcance global que el holismo semántico radical confiere a la variación del significado característica del paso de una teoría a otra inconmensurable.

La investigación que se presenta a continuación se elabora como respuesta a la demanda, abiertamente hecha por Kuhn, Feyerabend y por los impulsores de la corriente estructuralista, sobre la necesidad de analizar estructuralmente casos históricos de teorías inconmensurables para llegar a precisar formalmente y dar soporte empírico a la noción de inconmensurabilidad (Kuhn 1976 y Feyerabend 1977). Otra motivación general del trabajo es la de contribuir a una caracterización más coherente de la noción de inconmensurabilidad, desarrollando la pro-

\footnotetext{
${ }^{1}$ Este primer recurso a la noción de inconmensurabilidad se encuentra en Kuhn (1962/1970) y Feyerabend (1962).
} 
puesta del último Kuhn de presentar esta como un relación local o parcial entre teorías (Kuhn 1983, pp. 669-688), lo que permitiría superar las paradojas suscitadas por otras nociones más extremas. En particular, se pretende superar la paradoja consistente en afirmar que teorías globalmente inconmensurables se aplican, no obstante, a un dominio común.

Por lo que respecta al enfoque asumido en la investigación desarrollada aquí, su especificidad consiste en combinar el enfoque de tipo formal estructuralista con el enfoque de carácter informal historicista. Lo que constituye la novedad del enfoque no es, pues, otra cosa que la aplicación de un tratamiento formal a un caso concreto de inconmensurabilidad, teniendo en cuenta el contexto histórico de conocimientos y de creencias en el que surge dicho caso. ${ }^{2}$ Esta elección metodológica contrasta con la que subyace a la reconstrucción estructural del caso del flogisto ofrecida por Andreas Kamlah. A pesar de la rigurosidad y utilidad de su estudio, este no solo se revela como explícitamente anacrónico, ${ }^{3}$ sino también como limitado por su recurso a la distinción teórico/observacional, evitada por estructuralistas posteriores y reemplazada por aquella entre conceptos $\mathbf{T}$ teóricos y conceptos $\mathbf{T}$-no-teóricos.

Adoptando el enfoque señalado, trataré de defender la plausibilidad de la tesis de la inconmensurabilidad local introducida por el último Kuhn. Defenderé, pues, que el enfoque estructuralista permite establecer un criterio restrictivo del alcance de la inconmensurabilidad y ello, a su vez, una comparabilidad interesan-

\footnotetext{
${ }^{2}$ P. Lorenzano (2008) presenta un estudio muy similar al que aquí se ofrece, tanto en cuanto a los objetivos y el enfoque asumidos como en relación con las conclusiones alcanzadas. Además del enfoque estructuralista, existen otros de gran sofisticación e interés para abordar el problema de la inconmensurabilidad. Cabe destacar tres de ellos: el tratamiento cognitivista y computacional de Paul Thagard, desarrollado en su obra Conceptual Revolutions (1992), aquel otro a cargo de Larry Laudan, inaugurador del enfoque denominado 'problemsolving approach', caracterizado en Progress and Its Problems (1977), y el propuesto por Ilkka Niiniluoto en el marco de su realismo científico crítico, que expone en "Scientific Progress" (1980).

3 "I shall use a quite modern formulation for my purpose, and I shall disregard completely the fact that chemists in the eighteen century were not able to express their ideas in the same way. I still think that I do not miss the point by my anachronistic account. Furthermore I do not try to do justice to the historical facts. I just want to formulate a phlogiston theory which is the best I can think of and which explains as many chemical facts as possible. After all the problem at issue is not only an historical one but also a problem of logical analysis" (Kamlah 1984, p. 223). El tratamiento anacronista que Kamlah realiza de la relación entre la teoría del flogisto y la del oxígeno descansa de forma crucial en conceptos como los de peso molecular o peso atómico, que no se hallaban disponibles hasta que John Dalton desarrolló su teoría atómica a comienzos del siglo XIX. Así descrita, la relación entre dichas teorías rivales puede parecer más estrecha estructuralmente de lo que realmente es, al emplearse, para redefinir los conceptos teóricos de una teoría a partir de los de la otra, más información de la disponible en aquel período histórico. No olvidemos que, para poder entender las razones por las que finalmente se produjo la transición de la teoría del flogisto a la del oxígeno, es necesario proporcionar una descripción históricamente fiel de las dos teorías en competencia. Al hacer uso de nociones anacrónicas, Kamlah introduce implícitamente un trasfondo experimental claramente fuera de las posibilidades experimentales para identificar substancias disponibles en la época de Priestley y Lavoisier. Más concretamente, la distinción química entre átomos y moléculas no se estableció antes de Amedeo Avogadro, requiriendo la determinación experimental de los gases a distintas condiciones de temperatura y presión, algo extensamente desarrollado en la teoría atómica daltoniana. Más aún, la conclusión de Kamlah de que la teoría del flogisto es reducible a una teoría del oxígeno restringida, a pesar de sus discrepancias relativas a los postulados ontológicos, solo se mantiene si aceptamos como versión canónica de la primera una conforme a la cual al flogisto le es atribuible un peso atómico negativo (véase Kamlah 1984, p. 227). Esto, sin embargo, no parece adecuado, puesto que dicha atribución suele considerarse como apoyada en una hipótesis ad hoc surgida, a su vez, solo una vez que la teoría del flogisto se hallaba en un fase de crisis.
} 
te entre teorías inconmensurables. Dicha comparabilidad requerirá analizar tanto las coincidencias o equivalencias conceptuales como las divergencias e incompatibilidades conceptuales. Es decir, se intentarán determinar no solo los componentes inconmensurables sino también los componentes inconmensurables de las teorías del flogisto y del oxígeno.

Por lo que concierne a la estructura del trabajo, las partes a desarrollar serán las siguientes: en primer lugar, la aclaración acerca del tipo de entidad teórica que conformarían las teorías del flogisto y del oxígeno, en segundo lugar, la presentación de sus correspondientes reconstrucciones estructurales y, finalmente, el análisis de las relaciones interteóricas entre la teoría del flogisto y la teoría del oxígeno, mostrando cómo su modo de relacionarse satisface las condiciones establecidas en las definiciones de reducción ontológica débil y de inconmensurabilidad. Dado lo limitado del espacio disponible para el desarrollo de los distintos temas, se habrán de presuponer tanto una familiaridad suficiente con el episodio histórico referido como un conocimiento mínimo de la metodología estructuralista.

\subsection{Caracterización del tipo de entidad teórica que constituyen las teorías del flogisto y del oxígeno}

Teniendo en cuenta que el objetivo de las reconstrucciones modeloteóricas que se ofrecen a continuación es la comparación estructural de dos teorías inconmensurables, deberá aclararse y justificarse previamente cuál de las diversas nociones estructuralistas de teoría se aplicará en este caso.

Desde un enfoque estructuralista sincrónico se contemplan principalmente dos posibilidades: concebir las teorías como elementos teóricos o como redes teóricas (Balzer, Moulines \& Sneed 1987, pp. 167-177). Aunque mencionada como cuestión preliminar, el tipo de entidad teórica que representa la teoría del flogisto y la del oxígeno es una cuestión empírica que solo puede esclarecerse a posteriori. La labor empírica de reconstrucción se inició tratando de probar la hipótesis más simple: que sendas teorías constituyen elementos teóricos. Una vez confirmada tal hipótesis por las reconstrucciones ofrecidas en los apartados posteriores, trató de evaluarse la hipótesis más compleja de que las dos teorías de la combustión analizadas perteneciesen a un tipo de teoría también más compleja, representable en el estructuralismo como red teórica. ${ }^{4}$ En concreto se intentaron caracterizar FLO y OXG como especializaciones de las redes teóricas de los prin-

\footnotetext{
${ }^{4}$ En la literatura estructuralista la noción de red teórica se define estableciendo dos condiciones: 1) que existan un conjunto finito y no vacío de elementos teóricos TE y una relación de especialización $\sigma ; 2$ ) que la relación de especialización se restrinja al conjunto TE. Añadiendo nuevas condiciones a las dos anteriores resulta posible definir un tipo de red teórica más restringido. Se trata de la red teórica arbórea o árbol teórico, cuyas características específicas son: 1) la conexión entre todos los elementos teóricos que pertenezcan a TE, de modo que para cualquier par de elementos teóricos diferentes pertenecientes a TE se cumpla que o bien ambos son especializaciones de algún otro elemento teórico común, o bien ambos tienen alguna especialización en común; 2) la existencia, dentro de dicho conjunto, de un único elemento teórico básico, esto es, de un solo elemento teórico que no constituye una especialización de ningún otro elemento teórico del citado conjunto. Puesto que la definición de red teórica presupone la de especialización, deberán apuntarse, siquiera de pasa
} 
cipios, de la composición y de las reacciones químicas. ${ }^{5}$ Ninguna de estas hipótesis pudo corroborarse, habiendo una posible razón, de índole general, para ello, como es el hecho de que FLO y $\mathbf{O X G}$ pertenezcan a una etapa incipiente de la química, en la que tanto los ámbitos de aplicación como los aparatos conceptuales de las teorías se solapaban frecuentemente, sin que las redes teóricas hubiesen llegado todavía a desarrollarse. ${ }^{6}$

Se evaluó, todavía, una última posibilidad, a saber, la de caracterizar ambas teorías como elementos teóricos alternativos pertenecientes a holones teóricos sucesivos. Dentro del estructuralismo, la denominación de 'holón teórico' se reserva para aquellas "totalidades" teóricas o macro-unidades científicas generadas a partir de la interconexión de un grupo de teorías mediante vínculos interteóricos o leyes puente (Balzer, Moulines \& Sneed 1987, pp. 386-423, y Díez \& Moulines $1997 / 1999$, p. 365). Tales vínculos, representados mediante funciones parciales, tendrán una direccionalidad definida (no serán reversibles), conectarán a todos los elementos teóricos del dominio con al menos otro y serán transitivos. Las teorías relacionadas pueden constituir parte de una disciplina o incluso de varias. Este parece ser el caso, precisamente, de las dos teorías en estudio, ambas vinculadas no solo a teorías químicas como el camaralismo, sino también a teorías físicas como la mecánica newtoniana y aun a otras convergentes con la medicina, como sucede en FLO con la iatroquímica. ${ }^{7}$ El vínculo general característico de los ho-

da, los principales rasgos definitorios de esta. Son los siguientes: a) igualdad entre las clases de modelos potenciales y de modelos potenciales parciales de los respectivos elementos teóricos relacionados, y, b) inclusión de la clase de modelos actuales, la clase de condiciones de ligadura, la clase de vínculos y la de aplicaciones intencionales de la teoría que especializa respectivamente en la clase de modelos actuales, la clase de condiciones de ligadura, la clase de vínculos y la de aplicaciones intencionales de la teoría que es especializada. Expresado de otro modo, dos elementos teóricos relacionados mediante una relación de especialización compartirán su aparato conceptual, mientras que divergirán en cuanto al alcance de sus leyes y, consecuentemente, en cuanto a la extensión de sus clases de aplicaciones intencionales, ya que el elemento teórico que especializa restringe las leyes y el alcance empírico del elemento teórico especializado.

${ }^{5}$ En lo sucesivo, la teoría del flogisto se designará por medio de la abreviatura FLO, y la teoría del oxígeno mediante OXG.

${ }^{6}$ Como quedará patente en las reconstrucciones, ambos elementos teóricos divergen, en particular, en lo que atañe a su dependencia (FLO) o no (OXG) de la teoría de los principios y, por consiguiente, de una teoría de la composición química cualitativa. Ninguna de las dos teorías estudiadas constituye una red teórica porque no ha sido necesario establecer leyes más específicas para dar cuenta de diferentes tipos de casos concretos de combustión, ni es el caso que ambas teorías especialicen una misma teoría. Tampoco constituyen parte de una red teórica, puesto que no satisfacen ninguna de las condiciones que ha de cumplir toda especialización; es decir, ni las leyes de ninguno de los dos elementos teóricos restringe alguna ley fundamental establecida desde otro, ni sus correspondientes clases de los modelos potenciales y modelos potenciales parciales coinciden con los de algún otro elemento teórico. Como se verá en las reconstrucciones, $P$ y $Q$, por ejemplo, determinan respectivamente $\mathbf{M}_{\mathrm{p}}$ de $\mathbf{F L O}$ y de $\mathbf{O X G}$, pero no así $\mathbf{M}_{\mathrm{p}}$ de la teoría camaralista de los elementos (de la que dependen ambas). Del mismo modo, la relación de igual o menor respirabilidad, $R$, no determina $\mathbf{M}_{\mathrm{pp}}$ ni de la teoría de los principios, ni de la composición, ni de las reacciones y sí, en cambio, los $\mathbf{M}_{\mathrm{pp}}$ de las teorías de la combustión.

${ }^{7}$ La teoría química camaralista, de marcado carácter industrial, se desarrolló durante la segunda mitad del siglo XVII y comienzos del XVIII y sirvió como soporte teórico para las nociones de elemento y agregado presentadas en las teorías químicas del XVIII. En cuanto teoría de la composición, la química camaralista suministra un criterio para la clasificación de substancias, en virtud del cual a cada tipo de sustancia (fluida, gruesa y vidriosa) le corresponden uno o varios tipos de principios que le confieren su especificidad. De acuerdo con lo estipulado en esta teoría, ampliamente difundida por el camaralista Johann Becher, los elementos han de satisfacer las condiciones de pureza y simplicidad, considerándose la primera en el sentido de unicidad de 
lones teóricos puede adoptar la forma de cualquier tipo de relación interteórica (especialización, teorización, reducción, equivalencia) (Balzer, Moulines \& Sneed 1987, p. 390), por lo que en cada caso habrá que especificar su modo particular de concreción. Entra en juego, por tanto, un criterio mixto de identificación de totalidades teóricas. Aplicando el citado criterio al caso de estudio aquí tratado, se constata que la dependencia de las teorías de la combustión con respecto a otras obedece a que cada una de las primeras constituyen teorizaciones diferentes de las segundas. A pesar de no haber acometido la reconstrucción de estas, la última hipótesis apuntada parece suficientemente confirmada a partir de datos cruciales obtenidos en las reconstrucciones de las teorías rivales de la combustión.

Sin negar la importancia que el examen minucioso de este tipo de unidad macro-teórica tendría para la comprensión de la estructura global de disciplinas científicas como la química, ha de tenerse en cuenta que tal tarea rebasaría el propósito del presente análisis, a saber, el esclarecimiento del tipo de relación de inconmensurabilidad que se da entre FLO y OXG. Por otra parte, la restricción en el alcance de las reconstrucciones explica que no se hayan tomado en consideración conceptos centrales de la química de aquella época, como los de principio y elemento, característicos de otras teorías relacionadas con las teorías de la combustión. La exclusión de tales conceptos en las reconstrucciones comporta, como consecuencia, la omisión de toda referencia al cambio experimentado por ellos, habitualmente destacado por los teóricos de la inconmensurabilidad al tratar el caso del flogisto.

\section{Reconstrucción de la teoría del flogisto}

Por medio de FLO pretenden explicarse la combustión y otros fenómenos relacionados con ella como la calcinación, la reducción y la composición de sustancias químicas. ${ }^{8}$ Este conjunto de fenómenos de diversos tipos constituiría, pues,

\footnotetext{
estado de agregación y la segunda en el de indescomponibilidad en substancias distintas. Tanto el criterio como las condiciones aludidos se incorporan a la teoría del flogisto, asumiéndose aquel desde la teoría del oxígeno solo parcialmente; se adopta la taxonomía de substancias prescindiendo, en cambio, del aspecto relativo a los principios. Por otra parte, la distinción, también característica del camaralismo, entre compuestos y mezclas atendiendo respectivamente a la estabilidad e inestabilidad de la combinación cuantitativa de elementos, se recoge como clasificación máximamente general de los agregados tanto en la teoría del flogisto como en la del oxígeno (véase Partington 1962-1970, p. 639, y Brock 1992, p. 81). Por otra parte, varias nociones y principios específicos de la teoría iatroquímica, desarrollada principalmente durante comienzos del siglo XVII por continuadores del sistema de Paracelso como Joan-Baptista van Helmont y los defensores de la teoría ácido-alcalina, resultaron imprescindibles para definir el aire, la respirabilidad y la combustión dentro de las teorías del flogisto y del oxígeno. El estudio del aire fue llevado a cabo en gran medida por el iatroquímico Helmont, quien lo definió como un fluido elástico transportador de substancias. En la teoría del flogisto se recurrirá a la misma definición, que será contundentemente rechazada desde la teoría del oxígeno. Las nociones de respirabilidad y combustión, en la medida en que dependen del concepto de aire, se ven afectadas de la misma manera por la conexión con la teoría iatroquímica en el caso de la del flogisto y la desconexión con la misma teoría en el caso de la del oxígeno. No obstante, tanto la primera como la segunda comparten la vinculación con la teoría iatroquímica en lo concerniente a la caracterización de la respiración como emisión y absorción de gases (véase Brock 1992, pp. 47-54).

${ }^{8}$ Las principales fuentes historiográficas que se utilizarán en las reconstrucciones de las teorías del flogisto y del oxígeno son las siguientes: Conant (1948, pp. 67-115), Partington (1962), Cohen (1985) y Brock (1992). Aun-
} 
la clase de aplicaciones intencionales de FLO. Para dar cuenta de ellos se postulará la existencia de un principio químico, el flogisto, estableciéndose como ley fundamental la emisión de dicha sustancia en la combustión (por parte de las sustancias químicas sometidas a tal proceso).

Con objeto de simplificar el análisis metateórico, se tendrán en cuenta solo combustiones completas, es decir, combustiones en las que se agota el flogisto del cuerpo sometido a combustión. Por otra parte, todas las reacciones químicas consideradas serán combustiones, ocurriendo que cada modelo de FLO es una combustión. Antes de comenzar con las definiciones formales de los diferentes tipos de modelos, ha de enfatizarse que el presente estudio pretende ser fiel al contexto histórico en el que surgen las teorías; por lo que, con el fin de evitar incurrir en anacronismos, no se utilizarán ciertos términos cuantitativos (como 'densidad', 'volumen', etc.).

\subsection{Modelos potenciales de FLO}

$\mathbf{M}_{\mathbf{p}}(\mathbf{F L O}): x$ es un modelo potencial de la teoría del flogisto $\left(x \in \mathbf{M}_{\mathbf{p}}(\mathbf{F L O})\right)$ syss existen $S, T, C, A, F, c, w, R,{ }^{\circ}$ tales que ${ }^{9}$

(1) $x=\left\langle S, T, C, A, F, \mathbb{R}^{+}, c, w, R,{ }^{\circ}\right\rangle$

(2) $S$ es un conjunto finito no vacío

(3) $T=\left\{t_{1}, t_{2}\right\}$

(4) $C \subset S$ y $C \neq \varnothing$

(5) $A \subset S$ у $A \neq \varnothing$, asimismo $A \cap C=\varnothing$

(6) $F \subset S_{\text {y }} F \neq \varnothing$

(7) $c:\left(C \times A \times\left\{t_{1}\right\}\right) \rightarrow\left(S \times A \times\left\{t_{2}\right\}\right)$

(8) $w: S \rightarrow \mathbb{R}^{+}$

(9) $R \subseteq A \times A$, y $R$ determina un orden débil

(10) ${ }^{\circ}: S \times S \rightarrow S$

Interpretación pretendida básica: $S$ es un conjunto de porciones de sustancias químicas concretas (no tratándose de una clase de tipos de sustancias), $T$ es un conjunto cuyos únicos elementos son dos instantes temporales, uno anterior a la combustión $\left(t_{1}\right)$ y otro posterior a ella $\left(t_{2}\right)$. $C$ es un subconjunto de $S$ que excluye $A$, y se interpreta como el conjunto de porciones de sustancias combustibles concretas. $A$ es un conjunto de porciones de sustancias químicas de aire, esto es, de la sustancia que circunda los cuerpos sometidos a combustión. Finalmente, por lo que concierne a los dominios, $F$ es un conjunto de porciones de un elemento denominado 'flogisto'.

que rebasando los límites del estudio estrictamente histórico, las siguientes obras filosóficas de Philip Kitcher ofrecen un examen claro y detallado de la transición entre la teoría del flogisto y la del oxígeno en Kitcher $(1978,1982,1993)$. La obra de Paul Thagard de 1992, de carácter igualmente filosófico, ofrece asimismo un desarrollo histórico preciso del caso del flogisto. Lo mismo sucede con el artículo de Kamlah de 1984, a pesar de su planteamiento explícitamente anacronista. Cabe citar, por último, los trabajos de C.E. Perrin "The Chemical Revolution: Shifts in Guiding Assumptions" (véase bibliografía), Estany (1990).

${ }^{9}$ Siguiendo el modo de presentar las reconstrucciones utilizado en An Architectonic for Science, incluiré los conjuntos de números entre los constituyentes de los modelos. 
La función $c$ determina las combustiones o reacciones químicas, asignando a cada porción de sustancia combustible $\mathrm{c}$ y cada porción de aire a en $t_{1}$ una porción de sustancia $s$ y porción de aire $a^{\prime}$ en $t_{2}$. w es la función que determina el peso, asignando a cada sustancia química $s$ su peso $w(s)$ como un elemento de $\mathbb{R}^{+} . R$ relaciona diferentes porciones concretas de aire atendiendo a su igual o menor respirabilidad. Reparemos en que $R$ no tiene carácter funcional. Puede ocurrir que el mismo aire sea tan o menos respirable que más de un aire, esto es, que le correspondan varios aires tan o más respirables que él. La combinación de sustancias formando agregados se representa por medio de la función ${ }^{\circ}$. A cada par de sustancias se le asigna otra sustancia, un agregado, que resulta de la combinación de las dos primeras.

A continuación se definirán algunas nociones derivadas de los conceptos primitivos de FLO.

$$
\text { Si } x=\left\langle S, T, C, A, F, \mathbb{R}^{+}, c, w, R,^{\circ}\right\rangle \in \mathbf{M}_{\mathbf{p}}(\mathbf{F L O}) \text { entonces }
$$

(1) Sean $\left\langle c, a, t_{1}\right\rangle$ y $\left\langle s, a^{\prime}, t_{2}\right\rangle$ tales que $c\left(\left\langle c, a, t_{1}\right\rangle\right)=\left\langle s, a^{\prime}, t_{2}\right\rangle$, entonces $c^{1}(c)={ }_{\text {def }}$ $s$ y $c^{2}(a)=_{\text {def }} a^{\prime}$ (esto es, la imagen de una sustancia s bajo la función de combustión, $c^{1}(c)$, es el residuo de s obtenido a partir de s, y la imagen de una sustancia a bajo la función de combustión, $c^{2}(a)$, es la sustancia $a^{\prime}$ obtenida a partir de $a$ ).

En (1) se definen los conceptos de residuo de la sustancia combustible obtenido tras la combustión y de aire obtenido tras la combustión a partir del aire previo a la combustión. Tales conceptos se definen por medio de restricciones de $c$ respecto de los dominios $\mathrm{C}$ y $\mathrm{A}$.

(2) Sean $s, s^{\prime}, s^{\prime \prime}$ tales que ${ }^{\circ}\left(s, s^{\prime}\right)=s^{\prime \prime}$, entonces $s^{\prime \prime}={ }_{\text {def }} s^{\circ} s^{\prime}$ (léase $s^{\prime \prime}$ es la agregación de $s$ y $\left.s^{\prime}\right)$.

En (2) se define el concepto de agregado, mediante la aplicación de la función $\circ$, como el valor asignado a los argumentos de dicha función, esto es, como aquella sustancia resultante de la combinación de otras dos.

(3) $s$ contiene $s^{\prime}$ syss $_{\text {def }}$ existe algún $s^{\prime \prime}$ tal que $s=s^{\prime} \circ s^{\prime \prime}$ o $s=s^{\prime \prime} \circ s^{\prime}$.

En (3) se define la relación mereológica de contener, también a partir de la función ${ }^{\circ}$, como la relación entre el valor asignado a los argumentos de dicha función y uno u otro de éstos; es decir, como la relación entre aquella sustancia resultante de la combinación de otras dos y una u otra de éstas.

(4) $f_{\mathrm{s}}$ es la cantidad total (o máxima) del flogisto contenido en $s$ syss $_{\text {def }} s$ contiene $f_{\mathrm{s}}$ y para todo $f^{\prime}$ si s contiene $f^{\prime}$ entonces $f_{\mathrm{s}}$ contiene $f^{\prime}$.

A partir de la función de agregación y de la relación mereológica de contener (derivada de ella), resulta posible definir en (4) el concepto de cantidad total o máxima del flogisto contenido en una sustancia como aquella porción de flogisto que constituye uno de los argumentos de la función ${ }^{\circ}$ dando como valor $s$, ó, expresado en otros términos, como aquella porción de flogisto que se combina con alguna otra sustancia produciendo el agregado $s$.

(5) $P \subseteq S \times S$ y $s P s^{\prime}$ syss $_{\text {def }}: w\left(f_{s}\right) \leq w\left(f_{s}\right)$ (léase (aplicado a la combustión) $s^{\prime}$ se desflogistiza dando lugar a $s$ syss la cantidad total de flogisto de $s$ pesa menos o igual que lo que pesa la cantidad total de flogisto de $s^{\prime}$ ). 
$P$ se define en (5), a partir del concepto primitivo de peso y del concepto derivado de cantidad total de flogisto de una sustancia, como una relación entre diferentes porciones concretas de sustancias según su igual o menor contenido en flogisto. La relación $P$ determina un orden débil.

\subsection{Modelos de FLO}

M(FLO): $x$ es un modelo de la teoría del flogisto $(x \in \mathbf{M}(\mathbf{F L O}))$ syss existen $S, T$, $C, A, F, c, w, R,{ }^{\circ}$ tales que

(1) $x=\left\langle S, T, C, A, F, \mathbb{R}^{+}, c, w, R,{ }^{\circ}\right\rangle$

(2) $x \in \mathbf{M}_{\mathbf{p}}(\mathbf{F L O})$

(3) para todo $\left\langle c, a, t_{1}\right\rangle \in D_{I}(c)$ :

(3i) c contiene $c^{1}(c)$

(3ii) existe algún $f \in F$ tal que $s$ contiene $f$ en $t_{1}$ y $c^{1}(c)$ no contiene $f$ en $t_{2}$ y $c^{2}(a)$ contiene $f$ en $t_{2}$ y $a$ no contiene $f$ en $t_{1}$

(3iii) para todo $f \in F$ : si $a$ contiene $f$ en $t_{1}$ entonces $c^{2}(a)$ contiene $f$ en $t_{2}$

(4) para todo $a \in A: c^{2}(a) R a$ syss $w\left(f_{c(a)}^{2}\right) \geq w\left(f_{a}\right)$

La condición (3) es el requisito de que exista un tipo de entidad que actúe como principio en la combustión, siendo emitida por las sustancias sometidas a dicho proceso. (3i) requiere que la sustancia sometida a combustión contenga el remanente que quedará tras la combustión. (3ii) establece que la sustancia responsable de la combustión pase del cuerpo al aire. (3iii) exige que no pase nada de dicha sustancia del aire al cuerpo. Por último, en (4) se establece la disminución de la respirabilidad para aquella sustancia aire que, al haber absorbido flogisto, contiene una cantidad mayor de esta sustancia.

La demostración del siguiente teorema, implicado por los axiomas (3) y (4), permite concluir la menor respirabilidad del aire tras la combustión.

Teorema (I): para todo $\left\langle c, a, t_{1}\right\rangle \in D_{I}(c): c^{2}(a) R a$ y no es el caso que $a R c^{2}(a)$ Prueba: Supuesto $\left\langle c, a, t_{1}\right\rangle \in D_{I}(c)$ :

$\left\langle c^{1}(c), c^{2}(a), t_{2}\right\rangle=c\left(\left\langle c, a, t_{1}\right\rangle\right)$ por definición de $c$

para todo $f \in F$ si a contiene $f$ en $t_{1}$ entonces $c^{2}(a)$ contiene $f$ en $t_{2}$ por (3iii)

$c^{2}(a) R a$

existe algún $f \in F$ tal que $c^{2}(a)$ contiene $f$ en $t_{2}$ y $a$ no contiene $f$ en $t_{1}$ no para todo $f \in F$ es el caso que si $c^{2}(a)$ contiene $f$ entonces $a$ contiene $f$ no es el caso que $a R c^{2}(a)$ por (4)

q.e.d.

A partir del axioma (4) y de la definición (5) se sigue un nuevo teorema, en el que se establece la menor flogististización del aire más respirable y la mayor flogististización del aire menos respirable. Es decir, el teorema expresa una correlación entre la menor respirabilidad del aire que interviene en la reacción y su mayor flogististización, presentando la restricción de $P$ al dominio $A$ como la relación inversa de $R$, y, por tanto, como implicando una menor cantidad del flogisto en el aire más respirable. 
Teorema (II): para todo $\left\langle c, a, t_{1}\right\rangle \in D_{I}(c): a P c^{2}(a)$ syss $c^{2}(a) R a$

Prueba: Supuesto $\left\langle c, a, t_{1}\right\rangle \in D_{I}(c)$ :

$\left\langle c^{1}(c), c^{2}(a), t_{2}\right\rangle=c\left(\left\langle c, a, t_{1}\right\rangle\right)$ por definición de $c$

para todo $a, a^{\prime} \in A: c^{2}(a) R a$ syss $w\left(f_{c(a)}^{2}\right) \geq w\left(f_{a}\right)$ por (4)

$c P s$ syss $w\left(f_{\mathrm{c}}\right) \leq w(f)$ por def. (5)

$a P c^{2}(a)$ syss $w\left(f_{\mathrm{a}}\right) \leq w\left(f_{c(a)}^{2}\right)$ por restricción de $P$ al dominio $A$

$w\left(f_{a}\right) \leq w\left(f_{c(a)}^{2}\right) \operatorname{syss} w\left(f_{c(a)}^{2}\right) \geq w\left(f_{a}\right)$ por inversa de $\geq / \leq$

$a P c^{2}(a)$ syss $c^{2}(a) R a$ por sustitución a partir de def. (5) y axioma (4)

q.e.d.

\subsection{Modelos potenciales parciales de FLO}

$\mathbf{M}_{\mathbf{p p}}(\mathbf{F L O}): y$ es un modelo potencial parcial de la teoría del flogisto $(y \in$ $\left.\mathbf{M}_{\mathbf{p p}}(\mathbf{F L O})\right)$ syss existe un $x$ tal que $x=\left\langle S, T, C, A, F, \mathbb{R}^{+}, c, w, R,{ }^{\circ}\right\rangle$ $\in \mathbf{M}_{\mathbf{p}}(\mathbf{F L O})$ e $y=\left\langle S, T, C, A, F, \mathbb{R}^{+}, c, w, R,{ }^{\circ}\right\rangle$

La cuestión de la teoricidad atañe a $S, T, C, A, F, c, w, R$, ${ }^{\circ}$. Los dos primeros conjuntos básicos, junto con los dos siguientes derivados, la relación (no funcional) $R$ y las funciones, c, w, $y^{\circ}$, son FLO-no-teóricos, puesto que todos ellos pueden ser determinados sin recurrir a las leyes fundamentales de FLO. $F$ y la relación derivada, no-primitiva, $P$, en cambio, deben considerarse como FLO-teóricos dada su dependencia de dichas leyes. En particular, tanto la determinación de $F$ como la de $P$ exigen la asunción de la ley donde se afirma la existencia de un elemento, al que se denomina flogisto, que actúa como principio en la combustión. Tal ley aparece en la definición formal de $\mathbf{M}(\mathbf{F L O})$ como el axioma (3). A pesar de que flogisto y vocablos relacionales como flogistizar, desflogistizar, etc., sean los únicos términos FLO-teóricos, la importancia de los conceptos que se les asocian se refleja en el hecho de que necesariamente intervengan en la determinación de uno de los conjuntos derivados de la teoría. Dicha importancia se deriva del rol conceptual clave que ejercen articulando el esquema conceptual específico de FLO.

Examinaré pues, a continuación, los criterios para la determinación de las funciones y/o nociones expresadas por los términos FLO-no-teóricos. Los fenómenos o entidades a los que refieren dichos términos se reconocerán, al menos en parte, atendiendo a tales criterios. Ello queda especialmente patente en el caso de $S, A, c$ y $R$, al determinarse (durante el período histórico en que impera FLO) aplicando principalmente nociones independientes de toda teoría científica, pertenecientes al conocimiento ordinario.

Algunos términos del lenguaje ordinario son introducidos en las teorías científicas como términos primitivos, es decir, no definidos en dichas teorías. Así ocurre con la noción de combustión en FLO. Esta ha de entenderse como una noción primitiva únicamente determinable a partir de una disyunción de descripciones o predicados que expresan diferentes propiedades: aumento de temperatura, disminución de tamaño, emisión de gases, menor respirabilidad del 
aire circundante, ${ }^{10}$ etc. A partir de las razones ya apuntadas, es posible inferir que los criterios para la determinación de $c$ se identificarán con aquellos que se empleen en la determinación de $\mathbf{M}_{\mathbf{p p}}$. Pues, siendo la combustión el principal fenómeno del que trata FLO, y dado que la noción de combustión ha de caracterizarse dentro del lenguaje FLO-no-teórico (concretamente en el lenguaje ordinario, esto es, en el nivel más básico de conceptualización), se deduce que efectivamente la determinación de $c$ se rige por los mismos criterios que la de $\mathbf{M}_{\mathbf{p p}}$. $c$ es el fenómeno FLO-no-teórico que se pretende explicar.

Relegaré el análisis de los conceptos FLO-no-teóricos al apartado 2.5., donde se concretarán los vínculos interteóricos esenciales de FLO, es decir, aquellos vínculos necesarios para definir o caracterizar (en el caso de los términos primitivos) algunos de los conceptos FLO-no-teóricos. No obstante, adelantaré las distintas teorías o conceptualizaciones de que depende la determinación de tales conceptos. La función $w$ se determinará recurriendo a la mecánica clásica de partículas. La determinación de $T$ presupone algún tipo de teoría cronométrica. La teoría camaralista de la composición, junto con las teorías físicas de Boyle y Newton, resultarán imprescindibles en la determinación de la función de agregación ${ }^{\circ}$. Como se ha indicado más arriba, $S, A, c$ y $R$ serán determinados empleando fundamentalmente el lenguaje ordinario, si bien la caracterización de las nociones de aire, combustión y respirabilidad exigirá asimismo tener en cuenta tanto la teoría iatroquímica de la composición como la física y la química de Boyle. $T$ no se tratará en 2.5., pues, al ser una noción muy común en teorías científicas y no específica de la química, el análisis de sus vínculos interteóricos no presenta especial interés para el presente estudio.

Antes de pasar al siguiente apartado, conviene aclarar una última cuestión: se trata del problema que parece comportar la inclusión del subdominio FLO-teórico $F$ en el dominio FLO-no-teórico $S$. Podría pensarse que tal inclusión supone la necesaria determinación FLO-teórica de $S$ y, por lo tanto, su carácter FLOteórico. Sin embargo, el problema es solo aparente, ya que el dominio $S$ no se determina recurriendo necesariamente a sus distintos subdominios, sino a partir de ciertas propiedades FLO-no-teóricas que ha de tener cada elemento de dicho dominio (tener peso, determinado tamaño, etc.). ${ }^{11}$ En el caso del subdominio $F$, que históricamente se descubrió como vacío, su inclusión en el dominio de las sustancias se explicaría por la aceptación de que no en todos los casos las sustancias a las que los químicos pretenden referirse sean empíricamente determinadas, permitiéndose que su existencia se infiera a partir de ciertas propiedades FLO. no-teóricamente determinables (como por ejemplo, la mayor o menor respirabilidad del aire que circunda la sustancia sometida a combustión).

\footnotetext{
${ }^{10}$ La menor respirabilidad del aire circundante no remite a ninguna ley de FLO pues resulta posible identificar la combustión en virtud de otras propiedades.

${ }^{11}$ Por las mismas razones, la inclusión del subdominio OXG-teórico $O$ en el dominio OXG-no-teórico $S$ no implica la necesaria determinación $\mathbf{O X G - t e o ́ r i c a ~ d e ~} S$.
} 


\subsection{Condiciones de ligadura de FLO}

Como se verá, las distintas constricciones identificables en FLO, al igual que las identificables en OXG, no son propiamente de dichos elementos teóricos, sino heredadas o importadas de otras teorías, en particular de la mecánica newtoniana, con la que se hallan conectados por medio de vínculos interteóricos. Hay dos condiciones de ligadura naturales: identidad del peso y extensividad del peso. Las constricciones $\mathbf{C}_{1}$ y $\mathbf{C}_{2}$ son las mismas que se presentan en la mecánica clásica de choque relativamente a la masa. En $\mathbf{C}_{1}$ se exige que el peso de cualquier sustancia sea independiente del sistema en el que aparece dicha sustancia. $\mathbf{C}_{2}$ requiere que relativamente a alguna operación de agregación, representada por la noción básica ${ }^{\circ}$, el peso se comporte como aditivo bajo dicha relación. ${ }^{\circ}$ asigna a cada par de sustancias químicas otra sustancia química cuyo peso resulta de la adición de ambas sustancias.

$\mathbf{C}_{1}(\mathbf{F L O})$ : para todo $X: X \in \mathbf{C}_{1}(\mathbf{F L O})$ syss $\varnothing \neq \chi \subseteq \mathbf{M}_{\mathrm{p}} \mathbf{C}_{\mathbf{1}}(\mathbf{F L O})$ y para todo $x, y \in X$ y para todo $s$ : si $s \in S_{x} \cap S_{y}$ entonces $w_{x}(s)=w_{y}(s)$

$\mathbf{C}_{2}(\mathbf{F L O})$ : para todo $\chi: \chi \in \mathbf{C}_{2}(\mathbf{F L O})$ syss $\varnothing \neq \chi \subseteq \mathbf{M}_{\mathrm{p}}(\mathbf{F L O})$ y para todo $x$, $y, z \in X:$

(a) ${ }^{\circ}: \mathbf{S} \times \mathbf{S} \rightarrow \mathbf{S}$ donde $\mathbf{S}:=\cup\left\{S_{x} / x \in \mathbf{M}_{\mathbf{p}}(\mathbf{F L O})\right\}$

(b) para todo $s \in S_{x}, s^{\prime} \in S_{y}$ (si $s \circ s^{\prime} \in S_{z}$ entonces $w_{z}\left(s^{\circ} s^{\prime}\right)=w_{x}(s)+$ $\left.w_{y}\left(s^{\prime}\right)\right)$

La condición de ligadura global de FLO sería:

\section{$\mathbf{G C}(\mathbf{F L O})=\mathbf{C}_{1}($ FLO $) \cap \mathbf{C}_{2}($ FLO $)$}

El teorema demostrado a continuación, implicado por el axioma (3) y por $\mathbf{C}_{2}(\mathbf{F L O})$, establece la disminución de peso de todo cuerpo sometido a combustión.

Teorema (III): para todo $\left\langle c, a, t_{1}\right\rangle \in D_{I}(c): w\left(c^{1}(c)\right)<w(c)$

Prueba: Supuesto $\left\langle c, a, t_{1}\right\rangle \in D_{I}(c)$ :

$\left\langle c^{1}(c), c^{2}(a), t_{2}\right\rangle=c\left(\left\langle c, a, t_{1}\right\rangle\right)$ por def. $c$

$c$ contiene $c^{1}(c)$ por $(3 \mathrm{i})$

$w\left(s^{\prime} \circ c^{1}(c)\right)=w\left(s^{\prime}\right)+w\left(c^{1}(c)\right)$ por $\mathbf{C}_{2}(\mathbf{F L O})$

$w(c)=w\left(s^{\prime}\right)+w\left(c^{1}(c)\right)$ por sustitución a partir de $(3 \mathrm{i})$

$w\left(c^{1}(c)\right)<w(c)$

q.e.d.

\subsection{Vínculos interteóricos de FLO}

En lo que sigue examinaré los diversos vínculos que han de considerarse como constituyentes esenciales de FLO. El primero de ellos es el que provee a FLO de los valores de la función $w$. Estos se determinan por medio de varios instrumentos, a saber, balanzas analíticas, balanzas de brazos y dinamómetros. El uso de tal instrumental requiere asumir algún modelo de la mecánica del sólido rígido (RBM). Teniendo en cuenta que tal teoría es reducible a la mecánica clásica de 
partículas $(\mathbf{C P M})$, puede afirmarse que se precisa un vínculo $\mathbf{L}_{\mathbf{1}}$ entre $\mathbf{M}(\mathbf{R B M})$ (о $\mathbf{M}(\mathbf{C P M})$ ) y $\mathbf{M}_{\mathrm{p}}(\mathbf{F L O})$.

$$
\mathbf{L}_{\mathbf{1}}(\mathbf{F L O}) \subseteq \mathbf{M}(\mathbf{R B M}) \times \pi(\mathbf{R B M}, 1,2,5) \times \mathbf{M}_{\mathbf{p}}(\mathbf{F L O}) \times \pi(\mathbf{F L O}, 1,2,9)
$$

determinado por la siguiente condición: ${ }^{12}$

Para todo $x \in \mathbf{M}(\mathbf{R B M})$ y $x^{\prime} \in \mathbf{M}_{\mathbf{p}}(\mathbf{F L O})$ :

$\left\langle x,\left\langle P_{x}, T_{x}, m_{x}\right\rangle, x^{\prime},\left\langle S_{x}, T_{x}, w_{x},\right\rangle\right\rangle \in \mathrm{L}_{1}(\mathbf{F L O})$ syss hay $s \in S_{\mathrm{y}} t=T_{x} \cap T_{x^{\prime}}$ tal que

(a) $S_{x^{\prime}}=P_{x}$

(b) $w_{x}(s)=m_{x}(s)$

$P$ se interpreta como un conjunto de partículas, $T$ como un conjunto de instantes temporales y $m$ como la función para la determinación de la masa.

Además de $w$, existen otras funciones, relaciones y dominios (conjuntos básicos) FLO-no-teóricos determinados por vínculos esenciales concretos con otras teorías. Sin embargo, al tratarse de teorías precientíficas y/o no axiomatizadas, no se acometerá la reconstrucción estructural o definición formal de dichos vínculos interteóricos. Se indicará simplemente, de manera informal, en qué consisten los vínculos interteóricos que determinan $S, C, A, c, R,{ }^{\circ},{ }^{13}$ señalando el modo en que las nociones FLO-no-teóricas que intervienen en tal determinación dependen de ciertas teorías. Como ya se ha señalado más arriba, la caracterización de algunas de estas nociones involucra principalmente un tipo de conceptualización expresada en el lenguaje ordinario, no presentada en forma de teoría. Solo algún aspecto de la caracterización dependerá directamente de teorías precientíficas o científicas anteriores. Así ocurrirá con las nociones representadas por $C, c$ y $R$, las cuales demandarán un análisis tanto de los vínculos estrictamente interteóricos como de la ligazón con conceptualizaciones pertenecientes al conocimiento ordinario. Huelga decir que este conocimiento ordinario, transmitido a través del lenguaje ordinario, ha de relativizarse, del mismo modo que con las teorías, al período histórico bajo consideración. Examinaré a continuación los términos 'sustancia', 'aire', 'respirabilidad', 'combustión', 'sustancia' 'combustible', y 'agregación' siguiendo el orden en que se han mencionado.

En la determinación de $S$ se emplea la noción de materia característica de la teoría física o corpuscular de la materia. Durante finales del siglo XVII y principios del XVIII las teorías mecanicistas de Boyle y Newton, precursoras de la teoría atómica de Dalton, consolidaron una concepción de las sustancias en términos eminentemente físicos, en cuanto conglomerados de partículas o cuerpos. Con objeto de definir el término 'materia' se hace uso de cualidades geométricas y de los mecanismos de afinidad y repulsión, rechazándose, en cambio, la utilización de cualidades secundarias (temperatura, color) así como de las formas de la

\footnotetext{
${ }^{12}$ Los axiomas designados en $\pi(\mathbf{R B M}, 1,2,5)$ se corresponden con los establecidos en Balzer, Moulines \& Sneed (1987, p. 269), donde se define la clase de los modelos potenciales para la mecánica del sólido rígido.

${ }^{13}$ El análisis informal que sigue se apoya en el estudio histórico de la química presentado en Brock (1992, pp. 43-86).
} 
física aristotélica (Brock 1992, pp. 66, 75). Indiscutiblemente, el peso ha de considerarse como una propiedad esencial de la materia. En realidad, solo con el desarrollo de la mecánica newtoniana comenzó a diferenciarse entre materia y peso. Resulta obvio que tal asociación conduce necesariamente a la dependencia de la teoría del flogisto con respecto a la mecánica clásica de partículas, pues los métodos de determinación del peso presuponen el empleo de CPM o de alguna teoría reducible a ella como RBM (Balzer, Moulines \& Sneed 1987, pp. 53-54). Al comienzo del presente epígrafe se indica la conexión entre los modelos de RBM y los modelos potenciales de FLO en virtud del peso.

La determinación del conjunto básico A se realiza de acuerdo con los modos de determinación del aire estipulados en la teoría iatroquímica presentada por Joan-Baptista van Helmont (Brock 1992, p. 49). En ella, el aire se concibe como el entorno de las reacciones químicas y se define como un fluido elástico que transporta partículas, sustancias, elementos químicos. Los resultados de los estudios químicos de Boyle a este respecto ratifican los ofrecidos por Helmont (Brock 1992, p. 59).

La relación de igual o menor respirabilidad del aire se deriva de la propiedad del aire de ser respirable, siendo esta determinable por medio del conocimiento ordinario, por ejemplo a partir de las acciones de inspirar y espirar. No obstante, para definir el concepto de respirabilidad, indisociable del concepto de aire, ha de recurrirse nuevamente a la definición iatroquímica del término 'aire'. Algunos de los modelos de la teoría iatroquímica coincidirán necesariamente con algunos de los modelos potenciales de FLO debido, también, a que la concepción de la respiración como absorción y emisión de gases, junto con la correspondiente caracterización de la noción de gas, constituyen otra de las aportaciones de Helmont a la química de su tiempo. El subconjunto de las sustancias formado por los gases se determinaría pues conforme al análisis iatroquímico de estos, por medio del cual se establece que los gases son compuestos formados por aire y tierra o agua (Brock 1992, p. 51).

En cuanto a la noción de combustión, a pesar de que existen algunos vínculos constitutivos con otras teorías imprescindibles para determinar $c$, tales vínculos no contribuirán a formar un criterio de determinación, esto es, un conjunto de condiciones necesarias y suficientes para esta. También deberán tenerse en cuenta, como modos de determinación de $c$, la comprobación del aumento del calor, de la disminución del tamaño, de la emisión de gases, de la menor respirabilidad del aire circundante, etc. Todo ello involucra, principalmente, diversas parcelas del conocimiento ordinario. No obstante, en la medida en que el concepto de gas interviene en dicha determinación y este se considere científico, existirá al menos un vínculo esencial derivado de dicha intervención. Como ya se ha señalado al analizar la noción de respiración, los gases se consideraban, conforme a la teoría iatroquímica y a la química de Boyle, compuestos y/o mezclas ya de tierra y aire ya de agua y aire (Brock 1992, pp. 51, 71-72). En consecuencia, y dado que la determinación de la función c exige tanto la comprobación de que en el proceso de combustión se emiten gases, como de la menor respirabilidad 
del aire circundante a la reacción, el vínculo esencial de c con las citadas teorías se generará por una doble vía. En primer lugar, de forma más directa, por su dependencia de la noción de gas; en segundo lugar, de manera más indirecta, por su dependencia de la noción de respiración, a su vez directamente dependiente de la de gas.

El concepto de agregación, caracterizado mediante la función ${ }^{\circ}$, da cuenta de dos casos diferentes dentro de la teoría camaralista: el de los compuestos y el de las mezclas. Su origen es físico, pues se cimienta en las teorías físicas de Boyle y Newton (Brock 1992, pp. 55, 75). Conforme a ellas, la materia está compuesta de partículas organizadas jerárquicamente en grupos o racimos para formar mezclas o compuestos. Las primeras se caracterizarán por haberse constituido mediante una combinación cuantitativamente inestable de elementos. Las segundas, por el contrario, se definirán en virtud de una combinación cuantitativamente estable de elementos.

Deben señalarse, no obstante, algunas diferencias significativas entre el concepto físico y el químico de composición. Con el primero se alude a la relación de concatenación entre partículas, cuyo resultado sería la concatenación o agrupación de dichas partículas. Con el segundo, en cambio, se explica la relación de agregación entre sustancias químicas, que conduciría a la formación de un agregado. La diferencia esencial estriba en que un agregado no consiste en una mera agrupación de sustancias sino en una nueva sustancia obtenida a partir de otras distintas. Este aspecto diferenciador no se trata empleando conceptos específicos de ninguna teoría científica sino nociones propias del lenguaje ordinario. Así, en la descripción de dicho aspecto diferenciador solo intervienen términos ordinarios como 'sustancia', 'formación', etc. Finalmente, ha de subrayarse la necesidad de tomar en consideración tanto la significación física como la estrictamente química de la noción de agregación. Pues, aunque parte del significado del término 'agregación' dependa de nociones propias del lenguaje ordinario, otra parte fundamental precisa de nociones características de la física para su determinación. En concreto, entrarán en juego los conceptos físicos de 'masa' y 'conservación de la masa'. Por ello, parece razonable sostener que existe un vínculo interteórico esencial de ${ }^{\circ}$ con las teorías corpusculares de Boyle y Newton, de modo que la noción de composición de tales teorías resulta imprescindible para fijar el significado de agregación. Sin embargo, como ya se ha indicado, la especificación de este vínculo será necesaria pero no suficiente para dar el significado de dicho término, habiendo de tenerse en cuenta también el mencionado conocimiento ordinario.

Si se concede que las teorías físicas de Boyle y Newton son imprescindibles en la determinación de $w,{ }^{\circ}$ y $S$, con la teoría iatroquímica y la química boyleana, necesarias para determinar $A, c$ y $R$, entonces el vínculo global de FLO podría definirse como la intersección de todos los vínculos interteóricos de FLO.

\subsection{Elemento teórico de $\mathbf{F L O}$ y su aserción empírica}

$\mathbf{T}(\mathbf{F L O}):=\langle\mathbf{K}(\mathbf{F L O}), \mathbf{I}(\mathbf{F L O})\rangle$ en donde

$\mathbf{K}(\mathbf{F L O}):=\left\langle\mathbf{M}_{\mathrm{p}}(\mathrm{FLO}), \mathrm{M}(\mathrm{FLO}), \mathbf{M}_{\mathrm{pp}}(\mathrm{FLO}), \mathbf{G C}(\right.$ FLO $), \mathbf{G L}($ FLO $\left.)\right\rangle$ 
$\mathrm{e} \mathbf{I}(\mathrm{FLO}) \subseteq \mathbf{M}_{\mathrm{pp}}(\mathbf{F L O})$ es tal que

(1) $\mathbf{I}_{0} \subseteq \mathbf{I}(\mathbf{F L O})$ donde $\mathbf{I}_{0}$ es \{la combustión de sustancias inorgánicas no metálicas (madera, carbón), la calcinación del mercurio, la reducción y la composición del mercurio\}

(2) todos los miembros de $\mathbf{I}(\mathbf{F L O})$ son suficientemente similares a aquellos de $\mathbf{I}_{0}$.

Formulación de la aserción empírica de FLO: las aplicaciones intencionales pueden extenderse a un conjunto de modelos conectados entre sí por $\mathbf{G C}(\mathbf{F L O})$ que satisfacen las leyes y los vínculos.

Más concretamente, la aserción expresa que los miembros de $\mathbf{I}(\mathbf{F L O})$ que consisten en varias estructuras de sustancias $S$ como son el aire $A$ y las sustancias combustibles $C$ pueden extenderse a estructuras que contienen la sustancia flogisto $F$. Las estructuras han de cumplir las leyes y las condiciones de ligadura. Los miembros de $\mathbf{I}(\mathbf{F L O})$ consistirán en una estructura de instantes temporales $T$, con una serie de funciones $c$ para determinar las reacciones de combustión, funciones $w$ para el peso, así como ${ }^{\circ}$ para la agregación. Las relaciones $R$ y $P$ también intervienen dando cuenta de los miembros de $\mathbf{I}(\mathbf{F L O})$, pues establecen conexiones entre distintas sustancias respectivamente en virtud de la propiedad de ser igual o menos respirable y de acuerdo con su igual o menor flogististización. En cada reacción individual o proceso de combustión determinado por $c, T$ y $S$ (y, consecuentemente, sus subconjuntos $C, A, F$ ) junto con las otras funciones y relaciones mencionadas, deben satisfacer las leyes de FLO. Por otra parte, cada sustancia de $S$ será extensiva en al menos un miembro de $\mathbf{I}(\mathbf{F L O})$. La ristra de funciones $w$ debe asignar a cada sustancia el mismo peso en toda reacción química en $\mathbf{I}(\mathbf{F L O})$ donde intervenga.

\section{Reconstrucción de la teoría del oxígeno}

Con OXG, al igual que con FLO, se intenta dar cuenta del fenómeno de la combustión así como de otros fenómenos asociados a ella; principalmente, la calcinación, la reducción (análisis) y la composición (síntesis) de sustancias químicas. El conjunto formado por los (tipos de) fenómenos mencionados constituye la clase de aplicaciones intencionales de OXG. Para explicarlos se postulará la existencia de un nuevo elemento químico, el oxígeno, estableciéndose como ley fundamental la absorción de dicha sustancia en la combustión (por parte de las sustancias químicas sometidas a tal proceso).

\subsection{Modelos potenciales de $\mathbf{O X G}$}

$\mathbf{M}_{\mathbf{p}}(\mathbf{O X G}): x$ es un modelo potencial de la teoría del oxígeno $\left(x \in \mathbf{M}_{\mathbf{p}}(\mathbf{O X G})\right)$ syss existen $S, T, C, O, c, w, R,{ }^{\circ}$ tales que

(1) $x=\left\langle S, T, C, A, O, \mathbb{R}^{+}, c, w, R,{ }^{\circ}\right\rangle$

(2) $S$ es un conjunto finito no vacío 
(3) $T=\left\{t_{1}, t_{2}\right\}$

(4) $C \subset S$ y $\mathrm{C} \neq \varnothing$

(5) $A \subset S$ y A $\neq \varnothing$, asimismo $A \cap \mathrm{C} \neq \varnothing$, siendo también el caso que $A \cap$ $O \neq \varnothing$

(6) $O \subset S$ y $O \neq \varnothing$

(7) $c:\left(\mathrm{C} \times A \times\left\{\mathrm{t}_{1}\right\}\right) \rightarrow\left(S \times A \times\left\{\mathrm{t}_{2}\right\}\right)$

(8) $w: S \rightarrow \mathbb{R}^{+}$

(9) $R \subseteq A \times A$, y $R$ determina un orden débil

(10) ${ }^{\circ}: S \times S \rightarrow S$

Interpretación pretendida básica: $S$ es un conjunto de porciones de sustancias químicas concretas, $T$ es un conjunto cuyos únicos elementos son dos instantes temporales, uno anterior a la combustión $\left(t_{1}\right)$ y otro posterior a ella $\left(t_{2}\right)$. C es un subconjunto de $S$ que incluye elementos de A y que se interpreta como el conjunto de porciones de sustancias combustibles concretas. A es un conjunto de porciones de sustancias químicas de aire, esto es, de la sustancia constituida por porciones de elementos, algunos de cuales intervienen en los procesos de combustión. Por último, en lo que concierne a los dominios, $O$ es un conjunto de porciones de un elemento denominado oxígeno.

La función c determina las combustiones o reacciones químicas, asignando a cada porción de sustancia combustible $c$ y cada porción de aire a en $t_{1}$ una porción de sustancia $\mathrm{s}$ y porción de aire $a^{\prime}$ en $t_{2}$. w es la función que determina el peso, asignando a cada sustancia química $s$ su peso $w(s)$ como un elemento de $\mathbb{R}^{+} . R$ relaciona diferentes porciones concretas de aire atendiendo a su igual o menor respirabilidad. Téngase en cuenta que $R$, a diferencia del resto de las relaciones que figuran en la definición de $\mathbf{M}_{\mathbf{p}}(\mathbf{O X G})$, no tienen carácter funcional. Puede ocurrir que el mismo aire sea tan o menos respirable que más de un aire, esto es, que le correspondan varios aires tan o más respirables que él. La combinación de sustancias formando agregados se representa por medio de la función ${ }^{\circ}$. A cada par de sustancias se les asigna otra sustancia, un agregado, que resulta de la combinación de las dos primeras.

A continuación se definirán algunas nociones derivadas de los conceptos primitivos de OXG, de manera análoga a como se ha hecho en la reconstrucción de FLO.

Si $x=\left\langle S, T, C, A, O, \mathbb{R}^{+}, c, w, R,{ }^{\circ}\right\rangle \in \mathbf{M}_{\mathbf{p}}(\mathbf{O X G})$, entonces

(1) Sean $\left\langle c, a, t_{1}\right\rangle$ y $\left\langle s, a^{\prime}, t_{2}\right\rangle$ de los que $c\left(\left\langle c, a, t_{1}\right\rangle\right)=\left\langle t, a^{\prime}, t_{2}\right\rangle$, entonces $c^{1}(c)$ $={ }_{\text {def }} s$ y $c^{2}(a)=_{\text {def }} a^{\prime}$ (esto es, la imagen de una sustancia c bajo la función de combustión, $c^{1}(c)$, es el residuo de $s$ obtenido a partir de $c$, y la imagen de una sustancia $a$ bajo la función de combustión, $c^{2}(a)$, es la sustancia $a^{\prime}$ obtenida a partir de $a$ ).

En (1) se definen los conceptos de residuo de la sustancia combustible obtenido tras la combustión y de aire obtenido tras la combustión a partir del aire previo a la combustión. Tales conceptos se definen por medio de restricciones de $c$ respecto de los dominios $S$ y $A$. 
(2) Sean $s, s^{\prime}, s^{\prime \prime}$ tales que ${ }^{\circ}\left(s, s^{\prime}\right)=s^{\prime \prime}$, entonces $s^{\prime \prime}={ }_{\text {def }} s^{\circ} s^{\prime}$ (léase $s^{\prime \prime}$ es la agregación de $s$ y $s^{\prime}$ ).

En (2) se define el concepto de agregado, mediante la aplicación de la función ${ }^{\circ}$, como el valor asignado a los argumentos de dicha función, esto es, como aquella sustancia resultante de la combinación de otras dos.

(3) $s$ contiene $s^{\prime}$ syss $_{\text {def }}$ : existe algún $s^{\prime \prime}$ tal que $s=s^{\prime} \circ s^{\prime \prime}$ o $s=s^{\prime \prime} \circ s^{\prime}$.

En (3) se define la relación mereológica de contener, también a partir de la función ${ }^{\circ}$, como la relación entre el valor asignado a los argumentos de dicha función y uno u otro de estos; es decir, como la relación entre aquella sustancia resultante de la combinación de otras dos y una u otra de estas.

(4) $o_{\mathrm{s}}$ es la cantidad total (o máxima) del oxígeno contenido en $s$ syss $_{\text {def }}: S$ contiene $o_{\text {s }}$ y para todo $o^{\prime}$ si $s$ contiene $o^{\prime}$, entonces $o_{\text {s }}$ contiene $o^{\prime}$.

A partir de la función de agregación y de la relación mereológica de contener (derivada de ella), resulta posible definir en (4) el concepto de cantidad total o máxima del oxígeno contenido en una sustancia como aquella porción de oxígeno que constituye uno de los argumentos de la función ${ }^{\circ}$ dando como valor $s$, o, expresado en otros términos, como aquella porción de oxígeno que se combina con alguna otra sustancia produciendo el agregado $S$.

(5) $Q \subseteq S \times S$ y $s Q s^{\prime}$ syss $_{\text {def }}: w\left(o_{s}\right) \leq w\left(o_{s^{\prime}}\right)$ (léase (aplicado a la combustión) $s^{\prime}$ se desoxigena (pierde oxígeno) dando lugar a s syss la cantidad total de oxígeno de $s$ pesa menos o igual que lo que pesa la cantidad total de oxígeno de $s^{\prime}$ ).

$Q$ se define en (5), a partir del concepto primitivo de peso y del concepto derivado de cantidad total de oxígeno de una sustancia, como una relación entre diferentes porciones concretas de sustancias según su igual o menor contenido en oxígeno. La relación $Q$ determina un orden débil.

\subsection{Modelos de $\mathbf{O X G}$}

$\mathrm{M}(\mathrm{OXG}): x$ es un modelo de la teoría del oxígeno $(x \in \mathbf{M}(\mathbf{O X G}))$ syss existen $S$, $T, C, A, O, c, w, R,{ }^{\circ}$ tales que

(1) $x=\left\langle S, T, C, A, O, \mathbb{R}^{+}, c, w, R,{ }^{\circ}\right\rangle$

(2) $x \in \mathbf{M}_{\mathbf{p}}(\mathbf{O X G})$

(3) para todo $\left\langle c, a, t_{1}\right\rangle \in D_{I}(c)$ :

(3i) $a$ contiene $c^{2}(a)$

(3ii) existe algún $o \in O$ tal que $a$ contiene $o$ en $t_{1}$ y $c^{2}(a)$ no contiene $o$ en $t_{2}$ y $c^{1}(c)$ contiene $o$ en $t_{2}$ y $c$ no contiene $o$ en $t_{1}$

(3iii) para todo $o \in O$ : si $s$ contiene $o$ en $t_{1}$ entonces $c^{1}(c)$ contiene $o$ en $t_{2}$ (4) para todo $a \in A: c^{2}(a) R a$ syss $w\left(o_{c(a)}^{2}\right) \leq w\left(o_{a}\right)$

La condición (3) es el requisito de que exista un tipo de entidad que sea absorbida en la combustión por las sustancias sometidas a dicho proceso. (3i) requiere que la sustancia aire que participa en la combustión contenga el remanente que quedará tras la combustión. (3ii) establece que la sustancia responsable de la 
combustión pase del aire al cuerpo. (3iii) exige que no pase nada de dicha sustancia del cuerpo al aire. En (4) se establece la disminución de la respirabilidad para aquella sustancia aire con menor cantidad de oxígeno.

La demostración del siguiente teorema, implicado por los axiomas (3) y (4), permite concluir la menor respirabilidad del aire tras la combustión.

(I) Teorema: para todo $\left\langle c, a, t_{1}\right\rangle \in D_{I}(c): c^{2}(a) R a$ y no es el caso que $a R c^{2}(a)$

Prueba: Supuesto $\left\langle c, a, t_{1}\right\rangle \in D_{I}(c)$ :

$\left\langle c^{1}(c), c^{2}(a), t_{2}\right\rangle=c\left(\left\langle c, a, t_{1}\right\rangle\right)$ por definición de $c$

$a$ contiene $o$ en $t_{1}$ y $c^{2}(a)$ no contiene $o$ en $t_{2}$ por 3 (ii)

$c^{2}(a) R a$ por (4) y por def. (5)

no para todo $o \in O$ no es el caso que $a$ contiene $o$ en $t_{1}$ y $c^{2}(a)$ no contiene $o$ en $t_{2}$

no para todo $o \in \mathrm{O}$ es el caso que $a$ contiene $o$ en $t_{1}$ entonces $c^{2}(a)$ contiene $o$ en $t_{2}$

no es el caso que $a R c^{2}(a)$ por (4)

q.e.d.

A partir del axioma (4) y de la definición (5) se sigue un nuevo teorema, en el que se establece la menor oxigenación del aire menos respirable y la mayor oxigenación del aire más respirable. Es decir, el teorema expresa una correlación entre la menor respirabilidad del aire que interviene en la reacción y su menor oxigenación, presentando la restricción de $Q$ al dominio $A$ como una relación isomórfica con ' $R$ ', y, por tanto, como implicando una menor cantidad de oxígeno en el aire menos respirable.

(II) Teorema: para todo $\left\langle c, a, t_{1}\right\rangle \in D_{I}(c): c^{2}(a) Q a$ syss $c^{2}(a) R a$ Prueba: Supuesto $\left\langle c, a, t_{1}\right\rangle \in D_{I}(c)$

$\left\langle c^{1}(c), c^{2}(a), t_{2}\right\rangle=c\left(\left\langle c, a, t_{1}\right\rangle\right)$ por definición de $c$

para todo $a, c^{2}(a) \in A$, para todo $o \in O: c^{2}(a) R a$ syss $w\left(o_{c(a)}^{2}\right) \leq w\left(o_{a}\right)$ por (4)

$s^{\prime} Q s$ syss $w\left(o_{s^{\prime}}\right) \leq w\left(o_{s}\right)$ por def. (5)

$c^{2}(a) Q a$ syss $w\left(o_{c(a)}^{2}\right) \leq w\left(o_{a}\right)$ por restricción de $Q$ con respecto a $A$

$c^{2}(a) Q a$ syss $c^{2}(a) R a$ por sustitución a partir de definición (5) y de axioma (4) q.e.d.

\subsection{Modelos potenciales parciales de $\mathbf{O X G}$}

$\mathbf{M}_{\mathbf{p p}}(\mathbf{O X G}):$ y es un modelo potencial parcial de la teoría del oxígeno $(y \in$ $\left.\mathbf{M}_{\mathbf{p p}}(\mathbf{O X G})\right)$ syss existe un $x$ tal que $x=\left\langle S, T, C, A, O, \mathbb{R}^{+}, c, w, R,{ }^{\circ}\right\rangle$ $\in \mathbf{M}_{\mathbf{p}}(\mathbf{O X G})$ e $y=\left\langle S, T, C, A, \mathbb{R}^{+}, c, w, R,{ }^{\circ}\right\rangle$

La cuestión de la teoricidad afecta a $\left\langle S, T, C, A, O, c, w, R,{ }^{\circ}\right.$. 'Oxígeno', 'oxidación' y términos derivados como desoxigenación, constituyen los únicos términos OXG-teóricos, ya que solo ellos dependen de las leyes de $\mathbf{O X G}$ para la determinación de su significado. La determinación de $O$ y de la relación no-primitiva $Q$ requieren, en concreto, la asunción del axioma (3) perteneciente a la defi- 
nición formal de $\mathbf{M}(\mathbf{O X G})$, donde se expresa una de las leyes fundamentales de OXG. En dicha ley se afirma la existencia de un elemento, el oxígeno, que es absorbido por todos los cuerpos sometidos a un proceso de combustión. El papel de los conceptos de oxígeno y oxidación en la articulación del esquema conceptual específico de $\mathbf{O X G}$ es primordial dada su involucración en la conformación de las leyes fundamentales de dicha teoría. Los conceptos de oxígeno y oxidación intervienen en el esquema conceptual proporcionado por $\mathbf{M}_{\mathbf{p}}(\mathbf{O X G})$, que, a su vez, está presupuesto en aquel ofrecido por $\mathbf{M}(\mathbf{O X G})$.

El resto de los conjuntos, los básicos $(S$ y $T)$ y los caracterizados a partir de ellos $\left(C\right.$ y $A$ ), junto con la relación (no funcional) $R$ y las funciones $\left(c, w y^{\circ}\right.$ ), pueden considerarse $\mathbf{O X G}$-no-teóricos, pues resultan determinables sin recurrir al concepto OXG-teórico de oxígeno ni a las leyes fundamentales de OXG. El hecho de que el oxígeno figure como un componente del aire no conlleva que 'aire' deba concebirse como un término OXG-teórico, ya que la identificación de la sustancia aire resulta posible por medios independientes de tales leyes proporcionados desde la teoría iatroquímica. Según lo establecido en ésta, el aire se definiría como el fluido elástico transportador de partículas que actuaría como entorno en la combustión. Se comprueba que FLO y OXG comparten todos sus términos T-no-teóricos. La idea kuhniana de que los términos teóricos de una teoría podrían infectar a los no teóricos no se confirma a la luz de los resultados del análisis precedente. ${ }^{14}$ En el caso del tránsito de la teoría del flogisto a la del oxígeno, la determinación de los conceptos FLO-no-teóricos heredados por OXG no precisa de la intervención de las leyes fundamentales de $\mathbf{O X G}$, por lo que se mantienen como conceptos OXG-no-teóricos.

Teniendo en cuenta que los modelos potenciales parciales se determinan por medio de conceptos T-no-teóricos y que, según lo dicho, FLO y OXG comparten todos sus conceptos no teóricos, se concluye que $\mathbf{M}_{\mathbf{p p}}(\mathbf{F L O})=\mathbf{M}_{\mathrm{pp}}(\mathbf{O X G})$. La coincidencia por parte de ambas teorías en proporcionar una explicación para el fenómeno no teórico de la combustión, que ejemplificarán de la misma manera, junto con la asunción de que las clases de aplicaciones intencionales son subconjuntos de las clases de los modelos potenciales parciales, permite inferir que $\mathbf{I}(\mathbf{O X G}) \cap \mathbf{I}(\mathbf{F L O}) \neq \varnothing$. Esto equivale a decir que las clases de aplicaciones intencionales de OXG y FLO no son excluyentes sino que se solapan, cubriéndose total o parcialmente.

El análisis precedente permite llevar a cabo un estudio sobre las discrepancias y coincidencias entre las aplicaciones intencionales exitosas y no exitosas de ambas teorías. Semejante estudio debería dar cuenta del éxito de ambas teorías al explicar la menor respirabilidad del aire circundante a la combustión. Asimismo tendría que explicar el éxito de OXG, frente a FLO, al resolver la anomalía del aumento de peso de los metales calcinados, y su fracaso, también en contraste con FLO, al dejar irresuelto el problema de la (como luego se comprueba) aparente disminución de peso de las sustancias no metálicas sometidas a combustión.

${ }^{14}$ Kuhn (1983, p. 673) manifiesta esta preocupación. 
No me detendré a elucidar el modo en que se determinan las distintas nociones expresadas por los términos OXG-no-teóricos pues, al tratarse de nociones compartidas por FLO, su criterio de determinación ya ha sido examinado en el epígrafe 1.5 .

\subsection{Condiciones de ligadura de $\mathbf{O X G}$}

OXG comparte con FLO las condiciones de ligadura heredadas (no propias) $\mathbf{C}_{1}$ y $\mathbf{C}_{2}$ para la identidad y extensividad del peso. Recordemos que en $\mathrm{C}_{1}$ se exige que el peso de cualquier sustancia sea independiente del sistema en el que tal sustancia aparece. $\mathrm{C}_{2}$ requiere que relativamente a alguna operación de agregación, representada por la noción básica ${ }^{\circ}$, el peso se comporte como aditivo bajo dicha relación. ${ }^{\circ}$ asigna a cada par de sustancias químicas otra sustancia química cuyo peso resulta de sumar el peso de ambas sustancias.

No debe pasarse por alto, sin embargo, un punto de discrepancia crucial entre FLO y OXG. El punto de divergencia tiene que ver con el teorema III de la teoría del flogisto, implicado por (3) y $\mathbf{C}_{2}(\mathbf{F L O})$. En él se establece la disminución de peso de toda sustancia tras haber sido sometida a un proceso de combustión. Como se ha indicado, este teorema se deduce a partir de la constricción que estipula la aditividad de la agregación y de una de las leyes fundamentales de FLO según la cual toda sustancia emite flogisto al ser quemada. Dado que OXG comparte $\mathbf{C}_{2}$ con $\mathbf{F L O}$, pero no así la mencionada ley fundamental, la deducción del mismo teorema a partir de los axiomas de $\mathbf{O X G}$ resulta imposible. Una consecuencia importante se desprende de esto, a saber, que desde $\mathbf{O X G}$ no se da cuenta de la aparente disminución de peso que experimentan algunos cuerpos al ser sometidos a combustión. Solo se proporcionará una explicación para el aumento de peso de los metales y algunos minerales (como el azufre) al ser quemados. Conjugando $\mathbf{C}_{\mathbf{2}}(\mathbf{O X G}$ ) y el axioma (3) incluido en la definición formal de $\mathbf{M}(\mathbf{O X G})$, donde se establece la absorción de oxígeno por parte de los cuerpos que reaccionan en la combustión, puede obtenerse un teorema en el que se exprese el aumento de peso experimentado por las sustancias combustibles al ser quemadas. Debe repararse en que dicho teorema contradice al segundo teorema de FLO derivado a partir de $\mathbf{C}_{2}(\mathbf{F L O})$ y del axioma (3) perteneciente a la definición formal de $\mathbf{M}(\mathbf{F L O})$. Sirva este apunte para destacar cómo el aumento de peso de los metales constituye la principal anomalía a la que se enfrentaron, sin éxito, los defensores de la teoría del flogisto, y cómo la resolución de dicha anomalía desde la teoría del oxígeno requiere rechazar la teoría del flogisto, negando algunas de las consecuencias que se siguen de la ley fundamental de dicha teoría.

A continuación se dan las definiciones de las condiciones de ligadura de OXG, coincidentes, como ya se ha indicado, con las de FLO.

$\mathbf{C}_{\mathbf{1}}(\mathbf{O X G})$ : para todo $X: X \in \mathbf{C}_{1}(\mathbf{O X G})$ syss $\varnothing \neq X \subseteq \mathbf{M}_{\mathbf{p}}(\mathbf{O X G})$ y para todo $x$, $y \in X$ y para todo $s$ : si $s \in S_{x} \cap S_{y}$ entonces $w_{x}(s)=w_{y}(s)$.

$\mathbf{C}_{2}(\mathbf{O X G})$ : para todo $X: X \in \mathrm{C}_{2}(\mathbf{O X G})$ syss $\varnothing \neq X \subseteq \mathbf{M}_{\mathbf{p}}(\mathbf{O X G})$ y para todo $x, y, z \in X$ : 
(a) ${ }^{\circ}: \mathbf{S} \times \mathbf{S} \rightarrow \mathbf{S}$ donde $\mathbf{S}:=\cup\left\{\mathrm{S}_{x} / x \in \mathbf{M}_{\mathbf{p}}(\mathbf{O X G})\right\}$

(b) para todo $s \in S_{x}, s^{\prime} \in S_{y}\left(\operatorname{si} s^{\circ} s^{\prime} \in S_{z}\right.$ entonces $w_{z}\left(s^{\circ} s^{\prime}\right)=w_{x}(s)+$ $\left.w_{y}\left(s^{\prime}\right)\right)$

La condición de ligadura global de $\mathbf{O X G}$ sería:

\section{$\mathbf{G C}(\mathbf{O X G})=\mathbf{C}_{1}(\mathbf{O X G}) \cap \mathbf{C}_{2}(\mathbf{O X G})$}

El teorema demostrado a continuación, implicado por el axioma (3) y por $\mathbf{C}_{2}(\mathbf{O X G})$, establece el aumento de peso de todo cuerpo sometido a combustión.

(III) Teorema: para todo $\left\langle c, a, t_{1}\right\rangle \in D_{I}(c): w\left(c^{1}(c)\right)>w(c)$

Prueba: Supuesto $\left\langle c, a, t_{1}\right\rangle \in D_{I}(c)$

$\left\langle c^{1}(c), c^{2}(a), t_{2}\right\rangle=c\left(\left\langle c, a, t_{1}\right\rangle\right)$ por definición de $c$

$c^{1}(c)$ contiene o en $t_{2}$ y $c$ no contiene $o$ en $t_{1}$ por (3ii)

$w\left(c^{1}(c)\right)=w(c)+w(o)$ por $\mathbf{C}_{2}(\mathbf{O X G})$

$w\left(c^{1}(c)\right)>w(c)$

q.e.d.

\subsection{Vínculos interteóricos de $\mathbf{O X G}$}

Es posible identificar seis vínculos esenciales de OXG. De ellos, los relativos a $S$, $C, w \mathrm{y}^{\circ}$ son compartidos con FLO. Al tratarse de vínculos ya examinados me limitaré a indicar su procedencia, sin detenerme a analizarlos. Como ya se señalaba en el capítulo anterior para determinar los valores de la función $w$ se necesita asumir algún modelo de la mecánica clásica de partículas $(\mathbf{C P M})$. Es decir, se precisa un vínculo $\mathbf{L}_{1}$ entre $\mathbf{M}(\mathbf{C P M})$ y $\mathbf{M}_{\mathrm{p}}(\mathbf{O X G})$. Del mismo modo, con respecto a la determinación de $S$, se apuntaba la necesidad de recurrir a la noción de materia tal como se define en las teorías mecanicistas de Boyle y Newton, así como a la caracterización de $\mathrm{W}$ ofrecida en $\mathbf{C P M}$. Por último, ${ }^{\circ}$ conserva también sus vínculos, los cuales conectan $\mathbf{O X G}$ con la mecánica clásica, con la teoría camaralista y con la teoría corpuscular de Boyle. No ha de olvidarse su dependencia del conocimiento ordinario, ya descrita en el capítulo anterior.

Los conceptos de aire, respiración, combustión y sustancia combustible pierden algunos de sus vínculos con la teoría iatroquímica al igual que con la física de Boyle. No obstante, su determinación, principalmente dependiente del conocimiento ordinario, no variará, de modo que los cambios de creencias que justifican la desaparición de dichos vínculos no justifican, sin embargo, que dicha variación se considere como generando un cambio conceptual.

Incidiré en un par de aspectos históricos relativos a las alteraciones de los vínculos interteóricos. El primero de ellos es la evolución del concepto de aire (Brock 1992, pp. 124-127); el segundo, el papel de Boyle como impulsor de la revolución científica y precursor del atomismo químico de Dalton. Ambos temas se hallan parcialmente vinculados, pues el aire no solo deja de considerarse como entorno para pasar a considerarse como un agregado compuesto por una variedad de elementos activos en la combustión, sino que también se disocia completamente del estado en el que se presente. El aire deja de considerarse una sustan- 
cia sui generis, meramente portadora de partículas y pasiva en el proceso de combustión, para pasar a considerarse, pues, como una sustancia compuesta por diferentes tipos de elementos, entre los que figura el oxígeno, y activa en el proceso de combustión. Los conceptos de respiración, combustión y sustancia combustible, en la medida en que dependen del concepto de aire, también verán modificados algunos de sus vínculos interteóricos con la teoría iatroquímica y la física de Boyle. Para la determinación de los tres conceptos, con todo, continuará siendo fundamental el empleo del conocimiento ordinario. Los estados, en general, se disocian de los elementos, explicándose los primeros en virtud de cambios de temperatura o afinidades con otras sustancias, no a partir de principios químicos o propiedades intrínsecas de los elementos. Como resultado de ello, en la química de Lavoisier el aire se identificará con los gases (agregados de elementos), sin que se requiera que las sustancias denominadas como tales permanezcan necesariamente en estado gaseoso. Según lo dicho, los elementos se disocian tanto de los estados como de los principios. En consecuencia, las reacciones químicas, incluyendo la combustión, ya no se explicarán recurriendo a principios sino a relaciones proporcionales entre sustancias. La desaparición efectiva de la noción de principio en química no ocurrirá hasta el surgimiento de la teoría atómica de Dalton. No obstante, los defensores de OXG harán un uso meramente nominal de tal noción, variando el significado de 'principio' hasta hacerlo coincidir con el de 'elemento sistemáticamente activo en determinado tipo de reacciones'.

$\mathrm{Si}$, como se ha supuesto en el capítulo anterior, se concede que las teorías físicas de Boyle y Newton son imprescindibles en la determinación de $w,{ }^{\circ}$ y $S$, entonces el vínculo global de $\mathbf{O X G}$ podría definirse como la intersección de todos los vínculos interteóricos de $\mathbf{O X G}$.

\subsection{Elemento teórico de $\mathbf{O X G}$ y su aserción empírica}

$\mathbf{T}(\mathbf{O X G}):=\langle\mathbf{K}(\mathbf{O X G}), \mathbf{I}(\mathbf{O X G})\rangle$, en donde

$\mathbf{K}(\mathbf{O X G}):=\left\langle\mathbf{M}_{\mathrm{p}}(\mathbf{O X G}), \mathbf{M}(\mathbf{O X G}), \mathbf{M}_{\mathrm{pp}}(\mathbf{O X G}), \mathbf{G C}(\mathbf{O X G}), \mathbf{G L}(\mathbf{O X G})\right\rangle$ y $\mathbf{I}(\mathbf{O X G}) \subseteq \mathbf{M}_{\mathrm{pp}}(\mathbf{O X G})$ es tal que

(1) $\mathbf{I}_{0} \subseteq \mathbf{I}(\mathbf{O X G})$ donde $\mathbf{I}_{0}$ es \{la combustión de sustancias inorgánicas no metálicas (madera, carbón), la calcinación del mercurio, la reducción y la composición del mercurio\}

(2) todos los miembros de $\mathbf{I}(\mathbf{O X G})$ son suficientemente similares a aquellos de $\mathbf{I}_{0}$.

Formulación de la aserción empírica de OXG: las aplicaciones intencionales pueden extenderse a un conjunto de modelos conectados entre sí por $\mathbf{G C}(\mathbf{O X G})$ que satisfacen las leyes y los vínculos interteóricos.

La aserción expresa que los miembros de $\mathbf{I}(\mathbf{O X G})$, que consisten en varias estructuras de sustancias $S$ como son el aire $A$ y las sustancias combustibles $C$, pueden extenderse a estructuras de la sustancia oxígeno $O$. Las estructuras han de cumplir las leyes y las condiciones de ligadura. Los miembros de $\mathbf{I}(\mathbf{O X G})$ consistirán en una estructura de instantes temporales $T$, con una serie de funciones 
$c$ para determinar las reacciones de combustión, funciones $w$ para el peso, así como ${ }^{\circ}$ para la agregación. Las relaciones $R$ y $Q$ también intervienen dando cuenta de los miembros de $\mathbf{I}(\mathbf{O X G})$, pues establecen conexiones entre distintas sustancias respectivamente en virtud de la propiedad de ser igual o menos respirable y de acuerdo con su igual o menor oxidación. En cada reacción individual o proceso de combustión determinado por $c, T$ y $S$ (y, consecuentemente, sus subconjuntos $C, A, O)$ junto con las otras funciones y relaciones mencionadas, deben satisfacer las leyes de $\mathbf{O X G}$. Por otra parte, cada sustancia de $S$ será extensiva en al menos un miembro de $\mathbf{I}(\mathbf{O X G})$. La ristra de funciones $w$ debe asignar a cada sustancia el mismo peso en toda reacción química en $\mathbf{I}(\mathbf{O X G})$ donde intervenga.

\section{Análisis de la relación de inconmensurabilidad entre FLO y OXG}

En el presente apartado argumentaré en favor de la tesis, asumida por algunos estructuralistas, de que toda noción coherente de inconmensurabilidad implica la de reducción aplicativa (Díez \& Moulines 1997/1999, p. 459), e incluso, la de reducción ontológica, aunque propondré una versión modificada de esta, que no presuponga la de reducción teórica. Repárese en que, si la inconmensurabilidad se presenta como incompatible con cualquier tipo de relación de reducción, se debería entonces disponer de recursos alternativos a la reducción para darle sentido a la afirmación de que teorías inconmensurables no reducibles de ninguna manera dan cuenta, no obstante, del mismo ámbito de realidad. Sin embargo, no ha habido una propuesta suficientemente desarrollada, en este sentido, por parte de los defensores de la tesis de la inconmensurabilidad.

El episodio de inconmensurabilidad reconstruido en este trabajo mostrará la posibilidad de aplicar una reducción aplicativa u ontológica débil entre teorías inconmensurables, aun cuando la reducción exacta, aproximativa u ontológica tout court no sean aplicables. La noción de reducción ontológica débil o aplicativa no presupone el cumplimiento de las condiciones para una reducción estricta o teórica, sino solo el de las condiciones para una correspondencia entre [cierto subconjunto especialmente relevante de] las aplicaciones pretendidas de las teorías, cuyos dominios habrían de ser compartidos o correlacionables con estructuras relacionales de la otra teoría. La propuesta de este tipo de reducción responde, en parte, a consideraciones metodológicas (como es garantizar una noción coherente de inconmensurabilidad) y, en parte, al reconocimiento de la evidencia metateórica disponible (derivada del estudio de teorías inconmensurables). ${ }^{15}$

\footnotetext{
${ }^{15}$ La literatura relevante a este respecto aparece citada en Moulines (1984, pp. 69-70). Allí se mencionan numerosos pares de teorías entre las que se da una relación de reducción ontológica, siendo plausible suponer que ella implicaría alguna suerte de traducción entre dichas teorías en el ámbito de modelos potenciales parciales (al menos para el caso de la reducción ontológica homogénea, que se expondrá más adelante). Algunos
} 
La postura que mantendré puede sintetizarse en términos acuñados por Stegmüller (y empleados también por Moulines), diciendo que la inconmensurabilidad aquí contemplada será la teórica y no la empírica (Stegmüller 1979, § 11, y Moulines 1982, p. 207). Es decir, la relación de inconmensurabilidad afectará a la clase de los modelos potenciales de las teorías involucradas, sin extenderse a la clase de los modelos potenciales parciales (esto es, las estructuras que determinan las aplicaciones intencionales de las teorías). Esta última clase constituirá un ámbito susceptible de reducción.

\subsection{Relación de inconmensurabilidad teórica entre FLO y OXG}

Reproduciré, a continuación, la definición de inconmensurabilidad dada por J. A. Díez y C.U. Moulines en una obra reciente en la que se presentan algunos nuevos desarrollos del programa estructuralista. ${ }^{16}$

Sean $N, N^{*}$ dos redes teóricas distintas. Diremos que $N$ es suplantable (inconmensurablemente) por $N^{*}$ syss existen una relación $\rho \subseteq M_{p}^{*} \times M_{p}$ y un conjunto no-vacío $I_{a}$ tales que:

(1) $\rho$ no es efectivamente calculable.

(2) $\rho_{e}$ es función, es efectivamente calculable y $\operatorname{Rec}\left(\rho_{e}\right)=M_{p p}$.

(3) No existen $n$ conjuntos no vacíos $M_{1}^{*}, \ldots, M_{n}^{*}$ incluidos en $M_{0}^{*}$ tales que $\rho\left[M_{1}^{*} \cup \ldots \cup M_{n}^{*}\right] \subseteq M_{0}$.

(4) (i) $I_{a} \subseteq I_{0} \cap \rho_{e}\left[I_{0}^{*}\right]$ y (ii) para todo $y \in I_{a}\left(y \in \mathrm{r}\left[M_{0}^{*}\right] \wedge y \notin \mathrm{r}\left[M_{0}\right]\right)$. (Díez $\&$ Moulines 1997/1999, p. 459)

$\mathrm{Al}$ explicar la definición formal precedente, ambos autores inciden en un aspecto de máximo interés. Se trata de la distinción entre dos factores de inconmensurabilidad: uno, conceptual (referido en la condición (1), que afecta a los modelos potenciales y, por tanto, los aparatos conceptuales de las respectivas teorías) y otro, proposicional (referido en la condición (3), que afecta a los modelos actuales y, por consiguiente, a las leyes de las respectivas teorías). El primero determina la imposibilidad de establecer una correlación sistemática entre los modelos potenciales (y, por tanto, entre los conceptos básicos) de las dos teorías. El segun-

de los pares de teorías aludidos son los siguientes: la teoría planetaria de Kepler y la mecánica newtoniana de partículas, la óptica geométrica y la óptica ondulatoria, la termodinámica simple de los gases y la teoría cinética del gas, la mecánica newtoniana de partículas y la mecánica cuántica, la genética mendeliana y la biología molecular (véase Moulines 1984, pp. 61-62).

${ }^{16}$ La definición de suplantación teórica con inconmensurabilidad se establece en Díez \& Moulines (1997/1999, pp. 456-460). La razón por la que no se reproduce aquí la definición de inconmensurabilidad dada en Balzer, Moulines \& Sneed (1987, pp. 306-320), tiene que ver con su menor interés para el análisis de este caso particular. En el presente estudio de caso se precisa de una definición de inconmensurabilidad que pueda ser independiente de la de reducción teórica y, de este modo, se ajuste a todos los casos posibles de inconmensurabilidad, entre ellos los no susceptibles de reducción teórica, como el aquí tratado. Disponemos de una propuesta estructuralista más reciente y adecuada para definir la relación de inconmensurabilidad. Su carácter más global permite sustituir condiciones muy concretas pero poco esclarecedoras para el presente caso por otras más generales y reveladoras. En particular, posibilita prescindir del prerrequisito de la relación de reducción, recurriendo a la relación $\mathbf{T}$-no-teórica $\rho_{e}$ para establecer una conexión suficientemente significativa entre las teorías. 
do determina la imposibilidad de deducir las leyes fundamentales de la teoría suplantada a partir de las leyes de la suplantadora. Por otra parte, las condiciones (2) y (4) dan cuenta del carácter parcial de la inconmensurabilidad (o del grado de conmensurabilidad que implica), atendiendo, igualmente, a los dos factores citados. En (2) se determina la posibilidad de correlacionar sistemáticamente los modelos potenciales parciales (y, en consecuencia, los conceptos T-no-teóricos básicos) de cada teoría. Finalmente, (4) determina que el conjunto de anomalías de la teoría suplantada forme parte del conjunto de aplicaciones intencionales de ambas teorías, y que tales anomalías no sean subsumibles bajo las leyes de la teoría suplantada pero sí lo sean bajo las leyes de la teoría suplantadora.

Para demostrar que la relación entre FLO y OXG cumple la condición (1) es necesario demostrar que dicha relación no satisface la definición de reducción exacta. Atendamos, pues, primeramente, a las seis condiciones constitutivas de la definición de reducción establecidas en el programa estructuralista (Balzer, Moulines \& Sneed 1987, pp. 275-279). En primer lugar, se establece la condición de derivabilidad (D) de las leyes de la teoría reducida $\mathbf{T}$ a partir de las leyes de la teoría reductora $\mathbf{T}^{\prime}$ y a través de la mediación de $\rho$. En segundo lugar, se requiere que la reducción sea compatible $(\mathrm{C})$ con las condiciones de ligadura de las dos teorías. Una tercera condición es la compatibilidad (L) de la reducción con los vínculos de ambas teorías. La cuarta condición exige que cada modelo potencial de $\mathbf{T}$ se halle relacionado (sea traducible) $(\mathrm{T})$ mediante la reducción con algún modelo potencial de $\mathbf{T}^{\prime}$. En quinto lugar, se requiere la independencia (I), con respecto a la definición de $\rho$, de la derivación expresada en (D). Por último, como sexta condición se establece la conexión (IA) entre las aplicaciones intencionales de las dos teorías.

Reproduciré ahora (en castellano) la definición formal de reducción propuesta en el programa estructuralista:

Si $\mathbf{T}$ y $\mathbf{T}^{\prime}$ son elementos-teóricos idealizados, entonces $\rho$ reduce directamente $\mathbf{T}$ $a \mathbf{T}^{\prime}\left(\mathbf{T}^{\prime} \rho \mathbf{T}\right)$ syss
(1) $\rho \subseteq \mathbf{M}_{\mathbf{p}}^{\prime} \times \mathbf{M}_{\mathbf{p}}$
(2) $D_{I I}(\rho)=\mathbf{M}_{\mathbf{p}}(\mathrm{T})$
(3) para todo $x^{\prime}, x$ : si $\left\langle x^{\prime}, x\right\rangle \in \rho$ y $x^{\prime} \in \mathbf{M}^{\prime}$, entonces $x \in \mathbf{M}(\mathrm{D})$
(4) para todo $X^{\prime} \subseteq D_{I}(\rho)$ : si $X^{\prime} \in \mathbf{G C}^{\prime}$ entonces $\rho\left(X^{\prime}\right) \in \mathbf{G C}(\mathrm{C})$
(5) para todo $x^{\prime}, x$ : si $x^{\prime} \in \mathbf{G L}^{\prime}$ y $\left\langle x^{\prime}, x\right\rangle \in \rho$ entonces $x \in \mathbf{G L}(\mathrm{L})$
(6) para todo $y \in \mathbf{I}$ hay algún $y^{\prime} \in \mathbf{I}^{\prime}$ tal que $\left\langle y^{\prime}, y\right\rangle \in \rho_{e}$ (IA)

Fijémonos ahora en las dificultades que la definición estructuralista de reducción presenta en su aplicación al presente caso, donde quedará patente la imposibilidad de calcular la relación de reducción entre FLO y OXG. En primer lugar, el cumplimiento de la condición (3) es incompatible con el cumplimiento de la condición (4). La intervención del flogisto en la combustión, necesaria para el cumplimiento de (3), comporta consecuencias incompatibles con la intervención del oxígeno en el mismo fenómeno, ya que la condición de ligadura $\mathbf{C}_{2}(\mathbf{O X G})$ de la extensividad del peso, necesaria para que se cumpla (4), comporta que en 
un caso la sustancia combustible disminuya de peso y, en el otro, que la misma sustancia aumente de peso. El marco conceptual donde el oxígeno es la sustancia principalmente activa en el proceso de combustión, reconocible por ser absorbida por las sustancias que son sometidas a dicho proceso, y donde estas, a causa de $\mathbf{C}_{2}(\mathbf{O X G})$, habrán de aumentar de peso, resulta incompatible con aquel otro en el que la sustancia principalmente activa en la combustión es el flogisto, reconocible por ser emitido por las sustancias que se sometan a dicho proceso y donde estas, a causa de $\mathbf{C}_{\mathbf{2}}(\mathbf{F L O})$, habrán de disminuir de peso. Incongruencias similares surgirían al tratar de determinar desde OXG el concepto derivado desflogististización, perteneciente a FLO. Por las razones expuestas, las condiciones (3) y (4) no pueden satisfacerse conjuntamente y, puesto que ambas son condiciones necesarias para la reducción exacta, puede concluirse ya que esta no se dará.

Demostración:

Supongamos que (3) para todo $x^{\prime}, x$ : si $\left\langle x^{\prime}, x\right\rangle \in \rho$ y $x^{\prime} \in \mathbf{M}(\mathbf{O X G})$, entonces $x \in \mathbf{M}(\mathbf{F L O})$, y (4) para todo $X^{\prime} \subseteq D_{I}(\rho)$ : si $X^{\prime} \in \mathbf{G C}(\mathbf{O X G})$, entonces $\rho\left(X^{\prime}\right) \in$ GC(FLO)

(4) para todo $X^{\prime} \subseteq D_{I}(\rho)$ : si $X^{\prime} \in \mathbf{G C}(\mathbf{O X G})$, entonces $\rho\left(X^{\prime}\right) \in \mathbf{G C}(\mathbf{F L O})$ Supongamos que $X^{\prime} \in \mathbf{G C}(\mathbf{O X G})$

para todo $X^{\prime}$ si $X^{\prime} \in \mathbf{G C}(\mathbf{O X G})$ entonces para todo $s \in S_{x^{\prime}}, s^{\prime} \in S_{y^{\prime}}$ (si $s^{\circ}$ $s^{\prime} \in S_{z^{\prime}}$ entonces $\left.w_{z^{\prime}}\left(s^{\circ} s^{\prime}\right)=w_{x^{\prime}}(s)+w_{y^{\prime}}\left(s^{\prime}\right)\right)$, por definición de $\mathbf{C}_{\mathbf{2}}(\mathbf{O X} \mathbf{G})$ y de GC(OXG)

para todo $\left\langle c, a, t_{1}\right\rangle \in D_{I}(c): w\left(c^{1}(c)\right)>w(c)$, Teorema (III) de OXG

para todo $X$ si $X \in \mathbf{G C}(\mathbf{F L O})$, entonces para todo $s \in S_{x}, s^{\prime} \in S_{v}$ (si $s^{\circ}$ $s^{\prime} \in S_{z}$ entonces $\left.w_{z}\left(s^{\circ} s^{\prime}\right)=w_{x}(s)+w_{y}\left(s^{\prime}\right)\right)$, por definición de $\mathbf{C}_{\mathbf{2}}(\mathbf{F L} \mathbf{O})$ y de GC(FLO)

para todo $\left\langle c, a, t_{1}\right\rangle \in D_{I}(c): w\left(c^{1}(c)\right)<w(c)$, Teorema (III) de FLO

para todo $\left\langle c, a, t_{1}\right\rangle \in D_{I}(c): w\left(c^{1}(c)\right)\left\langle w(c)\right.$ y para todo $\left\langle c, a, t_{1}\right\rangle \in D_{I}(c)$ : no $w\left(c^{1}(c)\right)<w(c)$

no es el caso que $\rho\left(X^{\prime}\right) \in \mathbf{G C}(\mathbf{F L O})$

Si $X^{\prime} \in \mathbf{G C}(\mathbf{O X G})$, entonces no es el caso que $\rho\left(X^{\prime}\right) \in \mathbf{G C}(\mathbf{F L O})$

para todo $X^{\prime} \subseteq D_{I}(\rho)$ : si $X^{\prime} \in \mathbf{G C}(\mathbf{O X G})$, entonces $\rho\left(X^{\prime}\right) \in \mathbf{G C}(\mathbf{F L O})$ y $\mathrm{Si}$ $X^{\prime} \in \mathbf{G C}(\mathbf{O X G})$, entonces no es el caso que $\rho\left(X^{\prime}\right) \in \mathbf{G C}(\mathbf{F L O})$

No es el caso que (3) para todo $x^{\prime}, x:$ si $\left\langle x^{\prime}, x\right\rangle \in \rho$ y $x^{\prime} \in \mathbf{M}(\mathbf{O X G})$, entonces $x \in \mathbf{M}(\mathbf{F L O})$, y (4) para todo $X^{\prime} \subseteq D_{I}(\rho)$ : si $X^{\prime} \in \mathbf{G C}(\mathbf{O X G})$, entonces $\rho\left(X^{\prime}\right)$ $\in \mathbf{G C}(\mathbf{F L O})$, por absurdo.

No se cumple, tampoco, la condición (6), dado que hay aplicaciones intencionales exitosas de FLO que se pierden en la transición a OXG, aunque se recuperen más adelante en el desarrollo de la química. A pesar de que la condición que establece la conexión (IA) entre las aplicaciones intencionales de las dos teorías no es satisfecha en una interpretación literal, sí lo es en una versión más restringida en donde $\mathbf{I}$ e $\mathbf{I}^{\prime}$ se sustituyan, según corresponda, por $\mathbf{I}_{\mathfrak{a}}$, (con- 
junto de anomalías de que adolece la teoría del flogisto), $\mathbf{I}_{\mathbf{0}^{\prime}}$ (conjunto de aplicaciones paradigmáticas de la teoría del flogisto) y $\mathbf{I}_{0^{\prime}}$ (conjunto de aplicaciones paradigmáticas de la teoría del oxígeno). En efecto, aunque no para cada aplicación exitosa de FLO existe la correspondiente aplicación exitosa en OXG (recuérdese, por ejemplo, la combustión del hidrógeno), es el caso que existe un conjunto formado por aplicaciones pretendidas de ambas teorías tal que dichas aplicaciones no son subsumibles bajo las leyes de FLO y sí lo son bajo las de OXG. Expresada formalmente, la nueva versión de la condición (IA) sería la siguiente: ${ }^{17}$

$$
\mathbf{I}_{a} \subseteq \mathbf{I}_{0} \cap \rho_{e}\left[\mathbf{I}_{0^{\prime}}\right] \text { y para todo } y \in \mathbf{I}_{a}\left(y \in \mathbf{r}\left[\mathbf{M}_{0^{\prime}}\right] \wedge y \notin \mathbf{r}\left[\mathbf{M}_{0}\right]\right)
$$

donde ' $\mathbf{r}$ ' representa la función de "recorte" de conceptos $\mathbf{T}$-teóricos, que asigna modelos potenciales parciales a modelos potenciales o actuales constituidos añadiendo conceptos $\mathbf{T}$-teóricos a dichos modelos potenciales parciales.

Tras revisar qué condiciones definitorias de la reducción exacta cumple la relación entre FLO y OXG y cuáles incumple, se comprueba que solo es satisfecha la última condición (IA), aquella que involucra principalmente a las aplicaciones intencionales y, por tanto, al marco conceptual $\mathbf{T}$-no-teórico $\left(\mathbf{M}_{\mathrm{pp}}\right)$ común a ambas teorías en el que se caracterizan tales aplicaciones intencionales, entre ellas, las anomalías de FLO resueltas en OXG. En conclusión, la relación entre FLO y $\mathbf{O X G}$ no se adecua a una relación de reducción exacta, dado que no cumple cinco de las seis condiciones necesarias para esta, pero, al satisfacer la condición (IA), sí se adecua a un nuevo tipo de reducción ontológica, de carácter débil o aplicativo (que se caracterizará a continuación), y satisface una de las dos condiciones generales de la reducción exacta, a saber, la resolución de anomalías de la teoría que pretende reducirse. La irreductibilidad se debe, en consecuencia, al incumplimiento de la otra condición general de la reducción exacta, la derivabilidad de las leyes de la teoría que pretende reducirse a partir de las leyes de la teoría que se pretende reductora.

La condición (2) del definiens de la relación de inconmensurabilidad se cumple pues, como se sigue de las definiciones de $\mathbf{M}_{\mathrm{pp}}(\mathbf{F L O})$ y de $\mathbf{M}_{\mathrm{pp}}(\mathbf{O X G})$, a cada modelo parcial de OXG (que interviene en la relación de reducción), le corresponde un modelo parcial de FLO hasta agotar el dominio de los modelos parciales de FLO. Más aún, como hemos visto, cada modelo parcial de OXG se identifica con un modelo parcial de FLO.

Se cumple, asimismo, la condición (3) del definiens de la relación de inconmensurabilidad, ya que la correlación entre modelos actuales en imposible en todos los casos, debido a que la aplicación de las leyes y la satisfacción de las condiciones de ligadura en ambas teorías es incompatible. Es decir, la aplicación de las leyes y la satisfacción de las condiciones de ligadura en una teoría son incompatibles con la aplicación de las leyes y la satisfacción de las condiciones de ligadura en la otra teoría. La deducibilidad de las leyes de la teoría suplantada a partir de

\footnotetext{
${ }^{17}$ Como se reiterará más adelante, esta condición se presenta en Díez \& Moulines (1997/1999, p. 459), como una de las condiciones definitorias de la relación de inconmensurabilidad.
} 
las leyes de la teoría suplantadora es imposible en todos los casos dado el alcance general de dicha incompatibilidad.

Finalmente, se cumple también la condición (4) del definiens de la relación de inconmensurabilidad, puesto que el conjunto (interesante) de anomalías de la teoría suplantada contiene anomalías subsumibles bajo las leyes de la teoría suplantadora (y no bajo las de la teoría suplantada). Cualquier ejemplo de calcinación de metales constituye un caso en el que se cumple lo anterior.

\subsection{Conmensurabilidad empírica y reducción ontológica débil entre FLO y $\mathbf{O X G}$}

Volviendo a la cuestión de la conmensurabilidad empírica entre teorías teóricamente inconmensurables, cabe preguntarse por los tipos de relaciones interteóricas globales en las que ella se plasma en el presente caso.

En primer lugar, la convergencia empírica entre ambas teorías queda patente en su convergencia en la aproximación preteórica o las presuposiciones teóricas. Como se establece en su definición, la relación interteórica de teorización se caracteriza por la presencia de un tipo especial de vínculos determinantes, como son los vínculos interpretativos (Balzer, Moulines \& Sneed 1987, pp. 250-251, 278-279). La existencia de vínculos determinantes entre la teoría teorizada y la que teoriza constituye una condición presupuesta que ha de cumplirse para garantizar la presencia de vínculos interpretativos. Estos, por otra parte, deben satisfacer los requisitos de que los vínculos determinantes desemboquen siempre en modelos actuales de la teoría precedente (o subyacente) y de que no sean "reversibles", esto es, que no impliquen otro vínculo determinante en sentido inverso. La determinación semántica de los términos FLO-no-teóricos y OXG-noteóricos, ya sea referencial o definicional, presupone la aceptación de las leyes de teorías que subyacen a ambos elementos teóricos. Dado que FLO y OXG comparten todos sus conceptos no teóricos, las teorías presupuestas en su determinación o interpretación serán las mismas. La teoría del flogisto y la del oxígeno coinciden, por tanto, en teorizar (o, al menos, presuponer) las mismas teorías de la materia, la respiración y la composición.

En segundo lugar, ambas teorías son reducibles aplicativamente o mediante una reducción ontológica débil, como se verá un poco más adelante. Téngase en cuenta, primero, que, tal y como Moulines explicita, la estructura conformada a partir de los términos $\mathbf{T}$-no-teóricos constituye el "mundo exterior" a $\mathbf{T}$, mientras que la estructura correspondiente a los términos T-teóricos puede concebirse como el aparato específico de T para "ver el mundo" (Moulines 1991, p. 277). El primer tipo de subestructura se considerará como un modelo potencial parcial de $\mathbf{T}$ y jugará un papel decisivo en la interpretación y aplicación empíricas del modelo potencial en el que se incluya. Con carácter más general, la clase de los modelos potenciales parciales se identifica con la base de contrastación empírica o aplicación empírica de $\mathbf{T}$, denominando al subconjunto relevante de ellos "aplicaciones intencionales" de T. Por lo que respecta a FLO y OXG, y como se desprende de lo establecido anteriormente, ambas serían teorizaciones de la físi- 
ca de Boyle y de la de Newton (necesarias para determinar el dominio de las sustancias y las funciones de agregación y peso), al tiempo que de la iatroquímica y de la química boyleana (imprescindibles en la interpretación del dominio de las sustancias aire, la función de combustión y la relación de respirabilidad). Asimismo, a tenor de lo dicho, las subestructuras de FLO y OXG configuradas a partir de los términos no teóricos compartidos 'sustancia', 'sustancia' 'combustible', 'aire', 'agregación', 'peso', 'aire', 'combustión' y 'respirabilidad' conforman el vocabulario en el que se caracterizarían los modelos potenciales parciales comunes de ambas teorías, y, por ello, necesariamente intervendrían en la caracterización de la base de contrastación compartida.

La noción de reducción ontológica fue introducida en el contexto metateórico estructuralista por C.U. Moulines, con objeto de enriquecer el esquema de reducción exacta, que ha sido reproducido en el apartado anterior. En tal esquema se obviaría, a su juicio, el aspecto ontológico inherente a nuestra intuición acerca de la reducción. Moulines explica su propuesta en el artículo "Ontological Reduction in the Natural Sciences" (Moulines 1984), así como en el epígrafe III.3.6. de Pluralidad y recursión, "Conexiones ontológicas en la reducción de teorías" (Moulines 1991, pp. 264-274). Ella también entra en juego, aunque de forma implícita, en la definición de inconmensurabilidad dada dentro del programa estructuralista (Balzer, Moulines \& Sneed 1987, pp. 317-320). El interés de la noción de reducción ontológica radica, pues, en que permite identificar la relación entre los dominios de las respectivas teorías, asegurando así que la relación teórica de reducción que se establezca entre estas nos dé información acerca del mundo, de lo empírico.

La noción de reducción ontológica es de gran relevancia para el tratamiento del problema de la inconmensurabilidad, ya que permite concebir un nuevo tipo de reducción ontológica, de carácter aplicativo, que esclarezca ciertos aspectos de dicho problema. Es necesario comenzar por señalar que la reconstrucción de la correspondencia entre dominios característica de la reducción ontológica puede emplearse no solo para comprobar la adecuación empírica de la reducción interteórica, sino también con la finalidad de determinar la conexión empírica entre teorías que mantengan una relación interteórica distinta a la de reducción teórica. Por ejemplo, puede hacerse uso de ella para examinar el tipo y grado de conexión empírica existente entre teorías inconmensurables a nivel teórico. Este será, de hecho, el propósito del análisis que se ofrece a continuación, en el que se mostrará cómo la relación entre FLO y $\mathbf{O X G}$ cae bajo la definición de un nuevo tipo de reducción ontológica y, en consecuencia, cómo la inconmensurabilidad teórica (asociada a irreducibilidad teórica) conlleva conmensurabilidad empírica (asociada a reducción ontológica).

Fijémonos en la formalización de la relación entre los dominios básicos realizada por C.U. Moulines. Dado que la relación estructural entre los conjuntos básicos de diferentes teorías podrá alcanzar un alto grado de complejidad y que, además, comporta consecuencias directas para la relación estructural entre las respectivas relaciones y funciones tipificadas sobre dichos conjuntos básicos, conviene recordar primero la forma de las tipificaciones. Estas indicarán qué clase de 
argumentos toman las funciones o a qué clase de relatos se aplican las relaciones; lo harán estableciendo un procedimiento para construir los conjuntos correspondientes a tales funciones o relaciones a partir de conjuntos previamente dados, sobre los que se aplican repetidamente las operaciones conjuntistas de conjunto potencia y producto cartesiano (Balzer, Moulines \& Sneed 1987, pp. 6-7).

Siendo $\mathbf{T}$ y $\mathbf{T}^{\prime}$ dos teorías relacionadas mediante reducción, cada uno de sus respectivos modelos potenciales tendrán la forma:

$$
\begin{aligned}
& x=\left\langle D_{1}, \ldots, D_{n}, A_{1}, \ldots, A_{m}, r_{1}, \ldots, r_{p}\right\rangle \mathrm{y} \\
& x^{\prime}=\left\langle D_{1}{ }^{\prime}, \ldots, D_{v^{\prime}}{ }^{\prime}, \ldots, A_{1}{ }^{\prime}, \ldots, D_{w^{\prime}}{ }^{\prime}, r_{1}{ }^{\prime}, \ldots, r_{z^{\prime}}{ }^{\prime}\right\rangle
\end{aligned}
$$

$D_{i}$ (о $\left.D_{i}^{\prime}\right)$ son los conjuntos básicos, $A_{i}$ (o $A_{i}^{\prime}$ ) los conjuntos básicos auxiliares, y $r_{i}$ (o $\left.r_{i}^{\prime}\right)$ las funciones o relaciones que constituyen el modelo potencial. Para cada $r_{i}$ habrá una tipificación $\tau_{i}$ permitiendo establecer que:

$$
\begin{aligned}
& r_{i} \in \tau_{i}\left(D_{i 1}, \ldots, D_{i h}, A_{j 1}, \ldots, A_{j k}\right) \\
& \text { donde }\left\{D_{i 1}, \ldots, D_{i h}\right\} \subseteq\left\{D_{1}, \ldots, D_{n}\right\} \mathrm{y} \\
& \left\{A_{j 1}, \ldots, A_{j k}\right\} \subseteq\left\{A_{1}, \ldots, A_{m}\right\} \\
& \text { (y análogamente para cada } r_{i}^{\prime} \text { en } \mathbf{T}^{\prime} \text { ). }
\end{aligned}
$$

Se dirá que $\rho \subseteq \mathbf{M}_{\mathbf{p}}^{\prime} \times \mathbf{M}_{\mathbf{p}}$ es un vínculo reductivo ontológico entre $\mathbf{T}^{\prime}$ y $\mathrm{T}$ si, además de satisfacer la definición formal de reducción, "está compuesto en parte al menos de una conexión entre algunos de los $D_{i}$ y algunos de los $D_{i}^{\prime}$ y quizás también algunos de los $A_{j}^{\prime \prime}$ (Moulines 1991, p. 266). Estos últimos no se tendrán en cuenta en la definición formal debido a que pertenecen al ámbito de la matemática y no son específicos de las teorías empíricas. Apoyándose en una distinción informal de M. Spector entre reducción preservadora de dominio y reducción con eliminación de dominio (Spector 1978), Moulines establece una distinción formal entre reducción ontológica homogénea y reducción ontológica heterogénea. Ambos tipos corresponden a dos formas generales en las que pueden relacionarse los dominios de teorías distintas vinculadas reductivamente. Si los conjuntos básicos de la teoría reducida se relacionan por medio de una identidad total o parcial (inclusión propia) con los de la teoría reductora, las teorías se considerarán conectadas mediante una relación de reducción ontológica homogénea. Mientras que, si alguno de los conjuntos básicos de la teoría reducida se relaciona con alguno/s de la teoría reductora de modo tal que no implica identificación de elementos sino correspondencia biunívoca entre dominios, las teorías se estimarán relacionadas por medio de una reducción ontológica heterogénea. Las teorías conectadas mediante ambos tipos de vínculos de reducción ontológica mantendrían una relación de reducción ontológica mixta. Veamos la definición formal del primer tipo de reducción, aquella relevante para el presente caso.

Si $\mathbf{T}$ es reducible a $\mathbf{T}^{\prime}$ por medio de $\rho$, entonces: $\rho$ es un vínculo reductivo (ontológico) homogéneo de $\mathbf{T}$ a $\mathbf{T}^{\prime}$ syss:

Para todo $x \in \mathbf{M}_{\mathbf{p}}^{\prime} \mathbf{M}_{\mathbf{p}}, x^{\prime} \in \mathbf{M}_{\mathbf{p}}{ }^{\prime}$, si $\rho\left(x^{\prime}\right)=x$, entonces hay algún $i, j \in \mathbb{N}$ tal que $D_{i}[x]$ es un conjunto básico de $x, D_{j}\left[x^{\prime}\right]$ es un conjunto básico de $x^{\prime}$, y $D_{i}[x] \subseteq D_{j}\left[x^{\prime}\right]$ 
Siendo las teorías del flogisto y del oxígeno no-reducibles, resulta obvio que la condición presupuesta en las dos definiciones anteriores, la reducibilidad entre ambas teorías, no se cumplirá. Sin embargo, dejando aparte ese punto, tiene todavía sentido preguntarse por la satisfacción o no del resto de las condiciones definicionales, aquellas directamente expresadas en las condiciones. Como se ha puesto de relieve anteriormente, al demostrar que entre FLO y OXG no se daba una relación de reducción estricta $\rho$, entre ambas teorías se da, no obstante, un tipo especial de relación reducción (representable mediante ' $\rho_{a}^{\prime}$ ) al nivel de sus respectivas clases de aplicaciones intencionales paradigmáticas. Si en la definición de reducción ontológica se sustituye la precondición de que se dé una relación $\rho$ por otra que establezca que ha de darse una relación $\rho_{a}$, se conseguiría establecer un nuevo tipo de relación de reducción ontológica. Para definir este nuevo tipo de relación es necesario, pues, definir primero la relación $\rho_{a}$, de la que depende el carácter novedoso de aquél.

Si $\mathbf{T}$ y $\mathbf{T}^{\prime}$ son dos elementos teóricos idealizados, entonces $\rho_{a}$ reduce débil o aplicativamente $\mathbf{T} a \mathbf{T}^{\prime}\left(\mathbf{T}^{\prime} \rho_{a} \mathrm{~T}\right)$ syss existen una relación $\rho_{e} \subseteq \mathbf{M}_{\mathbf{p p}}^{\prime} \times \mathbf{M}_{\mathbf{p p}}$ y un conjunto no-vacío $\mathbf{I}_{a}$ tales que:

(1) $\rho_{e}$ es función, es efectivamente calculable y para todo $y, y^{\prime}:$ si $\left\langle y^{\prime}, y\right\rangle \in \rho_{e}$ entonces $y^{\prime}=y$

(2) (i) $\mathbf{I}_{a} \subseteq \mathbf{I}_{0} \cap \rho_{e}\left[\mathbf{I}_{0}^{\prime}\right]$ y (ii) para todo $y \in \mathbf{I}_{a}\left(y \in \mathrm{r}\left[\mathbf{M}_{0}^{\prime}\right] \wedge y \notin \mathrm{r}\left[\mathbf{M}_{0}\right]\right) ”$.

La relación entre FLO y OXG cumple la condición (1) en virtud de las definiciones de las clases de los modelos potenciales parciales de sendas teorías. El cumplimiento de la condición (2) por dicha relación ya ha sido comentada en el apartado anterior.

Comprobemos ahora que $\rho_{a}$ es un vínculo reductivo (ontológico) débil homogéneo de FLO a OXG.

Tenemos que FLO es reducible a OXG por medio de $\rho_{a}$, por las definiciones de $\mathbf{M}_{\mathrm{pp}}(\mathbf{F L O})$ y $\mathbf{M}_{\mathrm{pp}}(\mathbf{O X G})$, así como por el análisis de la relación entre las clases de aplicaciones intencionales de ambas teorías previamente desarrollado

y que para todo $y \in \mathbf{M}_{\mathbf{p p}}(\mathbf{F L O}), y^{\prime} \in \mathbf{M}_{\mathbf{p p}}(\mathbf{O X G})$, si $\rho_{a}\left(y^{\prime}\right)=y$, entonces hay un $S, S^{\prime}$ tal que

$S(y)$ es un conjunto básico de $y, S^{\prime}\left(y^{\prime}\right)$ es un conjunto básico de $y^{\prime}$,

y $S(y)=S^{\prime}\left(y^{\prime}\right)$, por las definiciones de $\mathbf{M}_{\mathbf{p p}}(\mathbf{F L O})$ y $\mathbf{M}_{\mathbf{p p}}(\mathbf{O X G})$

Asimismo, tenemos que FLO es reducible a OXG por medio de $\rho_{a}$

y que para todo $y \in \mathbf{M}_{\mathbf{p p}}(\mathbf{F L O}), y^{\prime} \in \mathbf{M}_{\mathbf{p p}}(\mathbf{O X G})$, si $\rho_{a}\left(y^{\prime}\right)=y$,

entonces hay un $T, T^{\prime}$ tales que

$T(y)$ es un conjunto básico de $y, T^{\prime}\left(y^{\prime}\right)$ es un conjunto básico de $y^{\prime}$,

y $T(y)=T^{\prime}\left(y^{\prime}\right)$, por las definiciones de $\mathbf{M}_{\mathbf{p p}}(\mathbf{F L O})$ y $\mathbf{M}_{\mathbf{p p}}(\mathbf{O X G})$

Se cumplen pues, para $\mathbf{O X G} \rho \mathbf{F L O}$, todas las condiciones del definiens de la reducción ontológica homogénea. Todos los conjuntos básicos de FLO, a saber, S y $T$, se hallan vinculados homogéneamente con los conjuntos básicos de $\mathbf{O X G}$, $S^{\prime}$ y $T^{\prime}$. 
El hecho de que la situación sea diferente para alguno de los conjuntos o dominios derivados se debe a que alguno de ellos depende de conceptos T-teóricos, para los cuales no existe, ni puede existir, equivalente en la otra teoría. Concretamente, me refiero a los dominios $F$ de FLO y $O$ de OXG. No ocurre tan solo que $F$ no resulte identificable con ningún conjunto o subconjunto derivado de OXG ni correlacionable con ninguna tipificación de OXG, sino también que cualquier intento de identificación o correlación semejante por medio de alguna modificación en $\mathbf{O X G}$ conduciría a incompatibilidades dentro de la propia teoría. La irreductibilidad ontológica de los conjuntos derivados $F$ y $O$, ambos dependientes de conceptos $T$-teóricos con implicaciones incompatibles, manifiesta uno de los rasgos definitorios de la inconmensurabilidad entre la teoría del flogisto y la teoría del oxígeno. Por lo que respecta al resto de los dominios derivados, $A$ y $C$ se encuentran homogéneamente vinculados con $A^{\prime}$ y $C^{\prime}$.

\subsection{Resolución de anomalías y progreso científico de FLO a OXG}

En tanto que relación comparativa o gradual, conmensurable por lo que respecta a las subestructuras de los modelos potenciales $\left(\mathbf{M}_{\mathrm{pp}}, \mathbf{I}\right)$, la inconmensurabilidad permite comparar el éxito de ambas teorías a partir de las aplicaciones intencionales compartidas que constituyen anomalías en FLO (consistentes en el aumento de peso de ciertas sustancias al ser quemadas) y no así en OXG. Luego existe un procedimiento objetivo (interteórico) para comparar el mayor o menor éxito de la teoría del oxígeno relativamente a la teoría del flogisto. La explicación del progreso científico en virtud de la relación entre las aplicaciones intencionales de las teorías rivales es desarrollada en detalle por C.U. Moulines, en su artículo "Is There Genuinely Scientific Progress?" (Moulines 2000). El autor argumenta que la tesis de la inconmensurabilidad, así como aquella de la carga teórica que la acompaña, es compatible con la comparabilidad epistemológica en el nivel de las aplicaciones intencionales, a la vez que con el progreso científico, evaluable a partir de dicha comparabilidad. Este, sin embargo, no se entendería ya como un saber más acerca de las mismas cosas, lo que presupondría una concepción conceptualmente lineal y veritativamente acumulativa del desarrollo científico que presentaría las teorías como conjuntos de enunciados referidos a cosas, sino como un saber más sobre algunos subdominios idénticos u homomorfos de aplicaciones intencionales paradigmáticas de especial relevancia epistemológica o pragmática. Esta concepción del progreso científico admite que se produzcan discontinuidades conceptuales o cambios parciales en los universos de discurso de las teorías en competencia, pero también exige una mínima continuidad entre algunos de ellos. Del mismo modo, la discontinuidad conceptual no permite pensar en una acumulación de verdades ligada a la transición entre teorías, aunque sí en una acumulación de problemas resueltos (o de problemas especialmente interesantes resueltos). Desde el estructuralismo se afirma la existencia de un crite- 
rio epistémico-pragmático interteórico para priorizar ciertas aplicaciones intencionales cuya posible extensión a los modelos resulte de especial interés en ese sentido. Lo dicho se corresponde con la condición (4) (i) de la segunda definición de inconmensurabilidad citada. El saber más acerca de las mismas cosas se sustituye por un saber mejor cómo solucionar los mismos problemas. El carácter altamente pragmático de las aplicaciones intencionales refuerza esta idea. La posibilidad de extender más aplicaciones intencionales o, simplemente, aplicaciones más interesantes, a los modelos de una teoría equivale a poder resolver más problemas (epistémico-pragmáticos) o problemas más interesantes. Moulines formaliza este último aspecto, más cuantitativo, del progreso científico del siguiente modo:

$$
\mathrm{S}=: \mathbf{I} \cap \mathbf{r}(\mathbf{M}){ }^{18}
$$

donde 'S' es el éxito de una teoría, I su clase de aplicaciones intencionales y $\mathbf{r}(\mathbf{M})$ la clase de los modelos potenciales parciales que forman parte de sus modelos. El éxito de una teoría se define, pues, en virtud de aquellas aplicaciones intencionales efectivamente extendibles a modelos de la teoría a la que pertenecen.

Para comparar el éxito de dos teorías rivales aplicando la definición anterior, estas tendrán que compartir al menos parte de sus aplicaciones pretendidas y, en consecuencia, de sus modelos potenciales parciales; ya que, de lo contrario, ambas teorías no podrían ser comparadas con respecto a la misma propiedad, esto es, su éxito en la aplicación de la teoría a los mismos fenómenos. Al compartir I y $\mathbf{M}_{\mathbf{p p}}$, FLO y OXG comparten el tipo de propiedad requerida para examinar el mayor o menor éxito de una de ellas con respecto a la otra. A pesar de que se precisarán varias décadas para la demostración experimental del mayor éxito de la teoría del oxígeno con respecto al de la teoría del flogisto, lo cierto es que en la primera existen muchas más aplicaciones paradigmáticas extendibles a modelos que en la segunda. Ejemplo de ello son todos los casos de calcinación de los metales. Expresado formalmente:

\section{$\mathrm{S}[\mathbf{F L O}] \subseteq \mathrm{S}[\mathbf{O X G}]$.}

De lo dicho hasta aquí se infiere que la decisión entre teorías científicas se rige por un criterio racional de éxito (resolución de ciertos problemas cognoscitivos y/o pragmáticos); es decir, que dicho criterio interteórico permite la determinación y la comparación del progreso científico entre teorías inconmensurables $\mathbf{T}$ no-teóricamente conmensurables). Se refuta, de este modo, la idea de que la tesis de la inconmensurabilidad va ligada a la defensa del irracionalismo en la evaluación de teorías, así como a un relativismo teórico absoluto.

\section{Conclusiones}

Subrayaré, a continuación, los tres principales resultados que el estudio de caso presentado ha permitido alcanzar:

\footnotetext{
${ }^{18}$ Moulines ofrece una formalización relativa a redes teóricas (Moulines 2000, p. 190), la presentada aquí, relativa a elementos teóricos, supone una versión simplificada de aquella.
} 
1) La aportación de un ejemplo en favor de la tesis de que existen teorías localmente inconmensurables, más en particular, teóricamente inconmensurables pero empíricamente conmensurables.

2) La demostración de la irreducibilidad estricta entre sendas teorías dada la incompatibilidad entre el cumplimiento de la condición de deducibilidad y el de la condición de mantenimiento de las condiciones de ligadura.

3) La posibilidad de reducción aplicativa y ontológica débil homogénea entre las dos teorías y, en consecuencia, la determinación del factor clave para explicar la objetividad del progreso que supone la transición entre teorías inconmensurables.

Bibliografía

Balzer, W., Moulines, C.U. y J.D. Sneed (1987), An Architectonic for Science. The Structuralist Program, Dordrecht: Reidel.

Brock, W.H. (1992), The Fontana History of Chemistry, Londres: Fontana Press.

Cohen, I.B. (1985), Revolution in Science, Harvard: The Belknap Press of Harvard University Press.

Conant, J.B. (1948), “The Overthrow of the Phlogiston Theory: The Chemical Revolution of 1775-1789”, en Conant, J.B. y L.K. Nash (eds.), Harvard Case Histories in Experimental Science, vol. I, Case 2, Cambridge, Massachusetts: Harvard University Press, pp. 65-115.

Díez, J.A. y C.U. Moulines (1997/1999), Fundamentos de Filosofía de la Ciencia, Barcelona: Ariel.

Estany, A. (1990), Modelos de cambio cientifico, Barcelona: Crítica.

Feyerabend, P.K. (1962), "Explanation, Reduction, and Empiricism”, en Feyerabend, P.K., Realism, Rationalism and Scientific Method. Philosophical Papers, Vol. I, Part 1, Chapter 4, Cambridge: Cambridge University Press, 1981, pp. 44-96.

Feyerabend, P.K. (1977), "Changing Patterns of Reconstruction", The British Journal for the Philosophy of Science 28: 351-369.

Kamlah, A. (1984), “A Logical Investigation of the Phlogiston Case”, en Balzer, W., Pearce, D. y H.-J. Schmidt (eds.), Reduction in Science, Dordrecht: Reidel, pp. 217-238.

Kitcher, P. (1978), "Theories, Theorists, and Theoretical Change", The Philosophical Review 87: 519-547.

Kitcher, P. (1982), "Implications of Incommensurability”, Philosophy of Science Association 2: 689-703,

Kitcher, P. (1993), The Advancement of Science. Science without Legend, Objectivity without Illusions, Oxford: Oxford University Press.

Kuhn, T.S. (1962/1970), The Structure of Scientific Revolutions, Chicago: The University of Chicago Press.

Kuhn, T.S. (1976), "Theory-Change as Structure-Change: Comments on the Sneed Formalism", Erkenntnis 10: 179-199. 
Kuhn, T.S. (1983), "Commensurability, Comparability, Communicability”, Philosophy of Science Association 2: 669-688.

Laudan, L. (1977), Progress and Its Problems: Toward a Theory of Scientific Growth, Berkeley, CA: University of California Press.

Lorenzano, P. (2008), "Inconmensurabilidad teórica y comparabilidad empírica: el caso de la genética clásica", Análisis Filosófico 28: 239-279.

Moulines, C.U. (1982), Exploraciones metacientificas, Madrid: Alianza Editorial, Madrid.

Moulines, C.U. (1984), "Ontological Reduction in the Natural Sciences", en Balzer, W., Pearce, D. y H. J. Schmidt (eds.), Reduction in Science, Dordrecht: Reidel, pp. 51-70.

Moulines, C.U. (1991), Pluralidad y recursión. Estudios epistemológicos, Madrid: Alianza Editorial.

Moulines, C.U. (2000), "Is There Genuinely Scientific Progress?", en Jonkisz, A. y L. Koj (eds.), On Comparing and Evaluating Scientific Theories, Poznan Studies in the Philosophy of the Sciences and the Humanities 72: 173-197.

Niiniluoto, I. (1980), "Scientific Progress", Synthese 45: 427-464.

Partington, J.R. (1962), A History of Chemistry, vol. III, Londres: Macmillan and Company Limited.

Perrin, C.E. (1988), "The Chemical Revolution: Shifts in Guiding Assumptions", en Donovan, A., Laudan, L. y R. Laudan (eds.), Scrutinizing Science. Empirical Studies of Scientific Change, Dordrecht: Kluwer Academic Publishers, pp. 105-124.

Spector, M. (1978), Concepts of Reduction in Physical Science, Philadelphia: Temple University Press.

Stegmüller, W. (1976), The Structure and Dynamics of Theories, New York: Springer-Verlag.

Stegmüller, W. (1979), The Structuralist View of Theories, New York: Springer-Verlag.

Thagard, P. (1992), Conceptual Revolutions, Princeton: Princeton University Press. 